\title{
Chemical tracers of Lusitanian amphorae kilns from the Tagus estuary (Portugal)
}

\author{
M.I. Dias ${ }^{a}, *$ M.I. Prudêncio a ${ }^{a}$ M.A. Gouveia ${ }^{a}$, M.J. Trindade ${ }^{a}$, R. Marques ${ }^{a}$, D. Franco ${ }^{a}$, J. Raposo ${ }^{b, c}$, \\ C.S. Fabião ${ }^{\mathrm{d}}$, A. Guerra ${ }^{\mathrm{d}}$ \\ a Instituto Tecnológico e Nuclear, Estrada Nacional, 10, 2686-953 Sacavém, Portugal \\ ${ }^{\mathrm{b}}$ Ecomuseu Municipal do Seixal, Serviço de Arqueologia, Núcleo da Quinta da Trindade, Av. MUD Juvenil, 2840-471 Seixal, Portugal \\ ${ }^{\mathrm{c}}$ Centro de Arqueologia de Almada, Apartado 603 (Pragal), 2801-602 Almada Codex, Portugal \\ ${ }^{\mathrm{d}}$ Departamento de História e Centro de Arqueologia, Faculdade de Letras, Universidade de Lisboa, Alameda da Universidade, P-1600-214 Lisboa, Portugal
}

\section{A R T I C L E I N F O}

\section{Article history:}

Received 16 June 2009

Received in revised form

5 November 2009

Accepted 6 November 2009

\section{Keywords:}

INAA

Roman Amphorae kilns

Provenance

Tagus river

Classe 20-21/Dressel 14

Classe 22-23/Almagro 50-51c

Lusitana $3 / 9$

\begin{abstract}
A B S T R A C T
In this paper, the characterization of Roman amphorae from the Porto dos Cacos (PC) and Quinta do Rouxinol (QR) workshops, in the Tagus estuary, dating to a period between the 1st and 5th century AD was carried out on the basis of instrumental neutron activation analysis data on 260 amphorae fragments, together with mineralogical compositional studies obtained by X-ray diffraction.

Special attention was devoted to the study of the Dressel 14. Almagro 50/51c and Lusitana 3/9 amphorae in an attempt to establish whether or not it is possible to establish any correlation between the composition and typology, and between and within the production centres studied.

A description of the geochemical patterns associated with each production centre was first carried out separately, followed by a discussion comparing the two centres situated in the same sedimentary basin, identifying diagnostic chemical tracers for each one; also, in certain cases, a relative correlation with the typology was achieved. Both the definition of reference groups and the attribution of amphorae to their workshop origin relied on the use of chemometric techniques for data structure analysis, coupled with geochemical data analysis, especially regarding trace element data and its geochemical behaviour and distribution according to the geological environment of the region.

This approach complements and reinforces the conclusions drawn from typological and archaeological analyses. Considering the two kiln sites studied, we may talk of the production of two types of Roman amphorae in the lower Tagus, with the establishment of compositional groups defined according to their corresponding chemical signatures. The products of the PC workshop are characterized by high concentrations of $\mathrm{Co}$, As and $\mathrm{U}$, and low concentrations of $\mathrm{Fe}, \mathrm{Zn}, \mathrm{Sb}, \mathrm{Rb}, \mathrm{K}$; QR workshop amphorae show instead higher concentrations of $\mathrm{Fe}, \mathrm{Sb}$, and also of $\mathrm{Rb}$ and $\mathrm{Zn}$, and lower amounts of $\mathrm{U}$.

These results make it possible to understand the crucial importance of the lower course of the Tagus River within the framework of the analysis of the economy of the estuary at the time. This, in turn, may lead to a better understanding of production and trade within Lusitania and also with other Roman provinces.
\end{abstract}

(c) 2009 Elsevier Ltd. All rights reserved.

\section{Introduction}

The most abundant artefacts found in any Roman-period archaeological site, and particularly in workshops, are fragments of pottery. Thus, the study of amphorae occupies a distinguished place in Roman ceramics research. Amphorae were the larger two-handled pottery containers of the Greek and Roman epochs,

\footnotetext{
* Corresponding author. Tel.: +351 219946222; fax: +351 219946185

E-mail address: isadias@itn.pt (M.I. Dias).
}

used for the storage and transportation of liquids (i.e. wine, olive oil), as well as other foodstuffs (i.e. fish sauce products, garum). Generally, amphorae provide greater insight into socio-economic relations, since they were principally used to transport food products. Transport amphorae were ordinary containers of commodities, so today they are rightfully considered to be important historical documents, representing the most tangible evidence of ancient trade that remains today. The knowledge of the chemical tracer(s) of each workshop may provide insight into ancient transport, trade routes, and manufacturing practices, and also contributes to revealing improvements or decline in technology, as 
well as in raw materials exploitation strategies, such as changes in the exploration of clay pits.

We conducted an interdisciplinary study on Roman amphorae manufactured during the period between the 1st and the 5th century AD at two of the best known prominent workshops among the lower Tagus potteries, the Porto dos Cacos - PC (Alcochete) and the Quinta do Rouxinol - QR (Seixal) potteries. The two production centres were located on the left bank of the Tagus River (Fig. 1). Because of their geographical position, and according to the types of amphorae found at the locations, these workshops were most probably associated chiefly with the preparation of garum and fish salted products, in the surrounding areas, namely Lisbon, Troia and Setúbal which were important centres of exportation of fish preserves. Moreover, previous work comparing some amphorae from these production centres with amphorae found at the industrial trading site of Correeiros (located in Lisbon) points to the use of $\mathrm{QR}$ amphorae at this fish salting industry (Dias and Prudêncio, 2007; Raposo et al., 2005).

The close connection between these ceramics production centres and areas related to the production and handling of fishbased products is obvious. The exploitation of marine resources in this basin is to be expected, and archaeological remains point to an intense occupation, with fish-salting facilities close to the river mouth and small peripheral units further away, and with pottery centres located upstream, upon the banks of the main river and its tributaries (Fabião, 2004, 2008; Raposo et al., 2005).

In this work, a larger number of samples from each production centre were studied; together with the previously analysed samples, they constitute a large dataset used to establish differences and similarities between the chemical compositions of amphorae from the QR and PC Roman workshops. Therefore, the

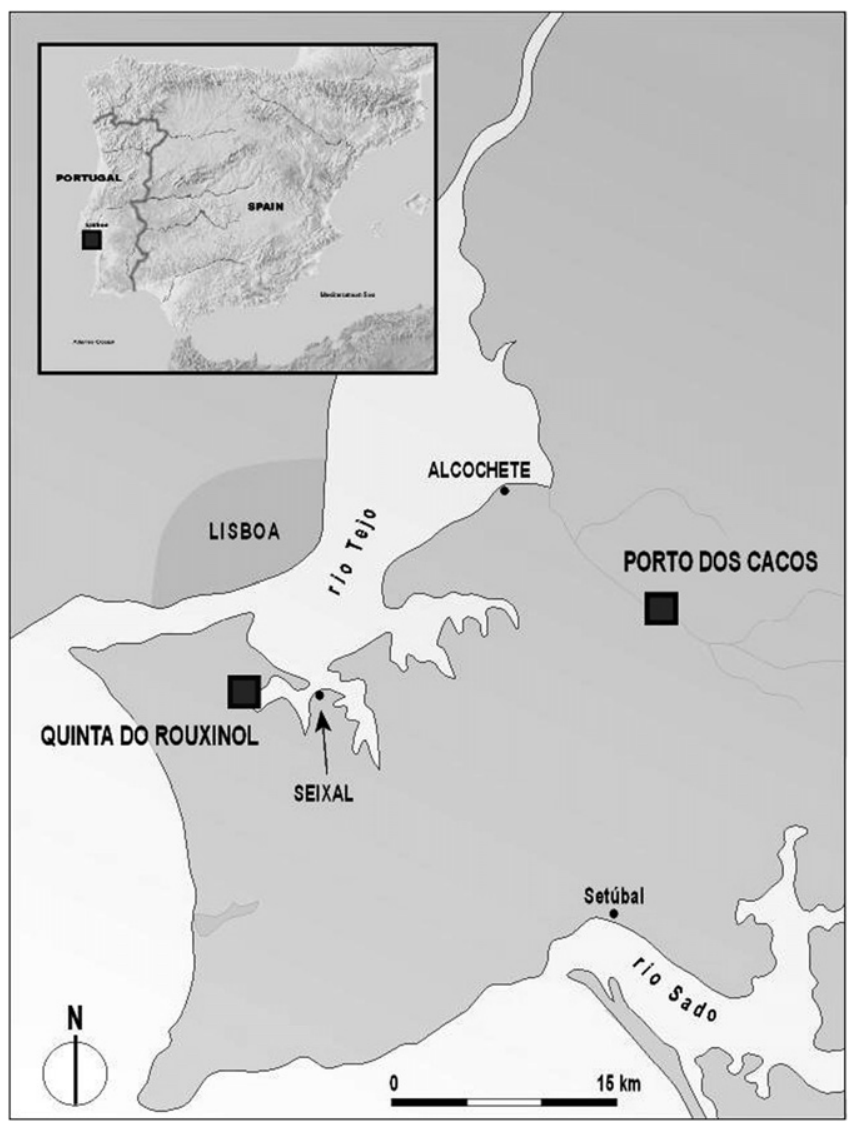

Fig. 1. Location of the Porto dos Cacos and Quinta do Rouxinol workshops. purpose of this paper is to study (i) whether all amphorae were manufactured with the same type of raw materials from the Tagus basin (ii) whether the presence of certain chemical elements in the ceramic pastes enables the differentiation and chemical tracing of each lower Tagus production centre,. This kind of approach will make available important geochemical patterns associated with the Roman production centres of the Tagus basin, to be used in further studies of provenance and in comparisons with consumption centres, thus providing significant information about the origin and development of industrial complexes and networks in Roman times.

Archaeometric studies of amphorae from the PC and QR Tagus production centres in comparison with a Sado basin production centre (Herdade do Pinheiro - HP) have already been performed, including a chemical characterization by instrumental neutron activation analysis (INAA) (Cabral and Gouveia, 1984; Cabral et al., 1993-1994, 1996, 2000, 2002; Prudêncio et al., 2003; Dias and Prudêncio, 2007). Some of these works emphasize the difficulty in distinguishing amphorae produced with similar raw materials from the Tertiary Tagus-Sado basin. Other works, including a petrographic identification of ceramic pastes from both basins (Mayet et al., 1996), also pointed to the difficulty in differentiating productions from the two estuaries (Sado and Tagus), due to the fact that they belong to a common Tertiary basin. Nevertheless, the application of adequate statistical treatment to a wide range of chemical analysis results has provided information that disclosed the relations among these production centres by classifying the samples into compositional groups. According to this data, subtle differences were found between them (Prudêncio et al., 2003; Raposo et al., 2005; Dias and Prudêncio, 2007) and within them as well (Dias et al., 2001). In addition, an assay on ceramic raw materials from the lower Tagus kilns has been carried out (Dias et al., 2003) by selecting some clay-supplying deposits from the surrounding area of each site (PC and QR). Chemical and mineralogical data obtained in the framework of that study emphasized the difficulty in establishing source materials of the same geological environment, especially estuarine ones, due to natural homogenization processes inherent to the transportation and deposition of those sediments. Even so, the results pointed to the use of two Mio-Pliocenic clays for amphora production at each site, and a certain similarity was also found between the iron content of $\mathrm{QR}$ amphorae and clays near that site.

More recently (Prudêncio et al., 2009), geochemical signatures of five Roman amphora production centres from the lower Sado have been established by the application of multivariate statistical analysis to chemical data obtained by INAA. Reference groups were identified, together with chemical indicators. Some of these sites are part of the same Tertiary Tagus-Sado basin.

Our intention in the present work is to complement the already existing information for the lower Sado basin, and also to provide chemical tracers for the most important Roman amphora production centres of the lower Tagus basin (Porto dos Cacos and Quinta do Rouxinol) identified so far, based on INAA. The methodology used emphasizes the encounter and complementarity between formal studies and chemical characterizations of pastes through the application of multivariate statistical methods necessary in order to quantify the similarities and differences between specimens and groups of specimens. Compositional groups are defined in accordance with the corresponding chemical signatures.

\section{The Roman amphora production centres from the lower Tagus basin}

The Porto dos Cacos (PC) archaeological site has been partially excavated in the late 1980s and two separate sets of kilns have been 
identified (Raposo, 1990; Raposo and Duarte, 1996). The first set featured a small kiln (kiln 1), which only maintained the lower part of its combustion chamber and the praefurnium. The second set included two other kilns found in close connection, and a possible third one, indicated by the geophysical survey. The second kiln (kiln 2) was completely excavated, showing a larger circular plant structure, with a furnace presenting a floor paved with small sandstone bricks and fragments of tiles. In addition, parts of the suspensurae and the grill they supported, together with parts of the vaulted baking chamber, could also be observed. The structure of kiln 3 was only partially excavated (Raposo and Duarte, 1996).

A significant amount of pottery was found at this site, such as domestic pottery, but especially amphorae, mostly used as containers for fish-based products, like the Class 20/21= Dressel 14. Class 22 =Almagro 50 and Class $23=$ Almagro 51c, and probably also for wine, like the Lusitana 3 and Lusitana 9. The entire set points to the abandonment of the PC production centre either at the end of the 4th century or during the first decades of the 5th century AD. It also indicates that Class $23=$ Almagro 51c and Class 22 =Almagro 50 amphorae were produced in kilns 1 and 2, the latter also producing the Lusitana 9 form. No identification of the kilns with the early production stages, represented by the Class 20/21 = Dressel 14 and Lusitana 3 forms, was possible, as these were only found in the dumping areas so far (Raposo et al., 1995). Among the sherds belonging to the latter form, it is possible to observe the largest and most significant series of potter markings known so far in Portugal - the cognomen Germanus is the most abundantly documented (Guerra, 1996). An interesting but enigmatic feature was found among the infrastructures that supported the use of the kilns, comprising a conspicuous row of 46 Class $20 / 21=$ Dressel 14 amphorae which are carefully placed vertically, defining an area whose function is still indeterminate.

The Quinta do Rouxinol (QR) production centre has also been excavated in the late 1980s (Duarte, 1990; Duarte and Raposo, 1996) and the excavations have revealed two kilns and the possible existence of a third one. The kilns are pear-shaped, and their only remains are the lower parts of the combustion chamber, the praefurnium and the bases of the suspensurae used to support the grills. Archaeological evidence points to a period between the middle of the 2 nd century AD and at least until the end of the 4th century AD when these kilns were functional, producing amphorae of Class 22 = Almagro 50, Class 23 = Almagro 51c and Lusitana 9 form, as well as domestic pottery.

\section{Experimental}

Amphorae fragments from both sites (PC and $Q R$ ), representative of the various typologies identified, have been chosen for chemical analysis. A total of 260 samples have been analysed distributed according to the typology for each site as described in Table 1. In the case of the PC workshop, most of the analysed sherds are samples of rims, and only 12 samples are fragments of bottom parts of the amphorae, six of A51c and six others of L3 typology.

The preparation for ceramic chemical analysis begins with the removal of soil or other surface materials from the sample. The procedure involves burring away the surface (inner and outer) with a tungsten carbide gouge, removing surface coatings and other impurities that may be attributed to contamination by weathering or other post-depositional processes. To the same purpose, the ceramic fragment is placed in deionised water for 24 hours, and afterwards for 30 more minutes in boiling clean deionised water. Next, ceramic fragments are dried in the oven at $80^{\circ} \mathrm{C}$, for a week. The interior paste from the ceramic fragment is then ground to a fine powder in an agate mortar, and homogenised. Aliquots of approximately $1 \mathrm{~g}$ each of powdered ceramics and reference materials are dried in an oven at $110{ }^{\circ} \mathrm{C}$ for 24 hours and stored in a desiccator. Once dried, 200-300 mg of powdered sample are weighed into clean polyethylene vials. Individual samples are prepared for long irradiations together with reference standard samples (GSD-9 and GSS-1) from the Institute of Geophysical and Geochemical Prospecting (IGGE). Reference values are obtained from the data tabulated by Govindaraju (1994).

One long irradiation and two gamma counts are performed. Samples and standards are bundled together in batches of 20 samples and 4 standards, and irradiated in the Portuguese Research Reactor pool for seven hours at a thermal flux of $3.34 \times 10^{12} \mathrm{n} \mathrm{cm}^{-2} \mathrm{~s}^{-1} ; \varphi_{\text {epi }} / \varphi_{\text {th }}=1.4 \% ; \varphi_{\text {th }} / \varphi_{\text {fast }}=12.1$. The bundles are rotated continuously during irradiation, to ensure that all samples receive the same neutron exposure. Iron (Fe) flux monitors are irradiated with the samples to allow corrections due to variation in the neutron flux. Two gamma-ray spectrometers are used: (1) one consisting of a $150 \mathrm{~cm}^{3}$ coaxial Ge detector connected through a Canberra 2020 amplifier to an Accuspec B (Canberra) multichannel analyser. This system has a FWHM of $1.9 \mathrm{keV}$ at $1.33 \mathrm{MeV}$; and (2) the other, consisting of a low energy photon detector (LEPD) connected through a Canberra 2020 amplifier to an Accuspec B (Canberra) multichannel analyser. This system has a FWHM of $300 \mathrm{eV}$ at $5.9 \mathrm{keV}$ and of $550 \mathrm{eV}$ at $122 \mathrm{keV}$. After a four-day decay, the samples are counted for 30 minutes in the high energy detector and 60 minutes in the low energy detector to measure medium-lived elements, including $\mathrm{Na}, \mathrm{K}, \mathrm{Ga}, \mathrm{As}, \mathrm{Br}, \mathrm{La}, \mathrm{W}$, $\mathrm{Sm}$ and U. Following an additional four-week decay, the samples are counted again for 2 hours and 30 minutes in the low and high energy detectors, yielding the measurement of the following long-lived elements: Fe, Sc, Cr, Co, Zn, Rb, Sb, Cs, Ba, Zr, Ce, Nd, Eu, $\mathrm{Tb}, \mathrm{Yb}, \mathrm{Lu}, \mathrm{Hf}$, Ta and Th. Corrections were carried out for spectral interference from uranium fission products in the determination of barium, rare earth elements (REE) and zirconium (Gouveia et al., 1987; Martinho et al., 1991). Relative precision and accuracy are, in general, within $5 \%$, and occasionally within $10 \%$.

The mineralogical composition was obtained by X-ray diffraction (XRD), using a Philips X'Pert Pro diffractometer, with a PW $3050 / 6 x$ goniometer, $\mathrm{Cu} \mathrm{K} \alpha$ radiation, and fixed divergence slit, operating at $45 \mathrm{kV}$ and $40 \mathrm{~mA}$. The powdered samples were prepared as non-oriented aggregates and used to obtain the diffraction patterns. Scans were run from 3 to $70^{\circ} 2 \theta$, using a step size of $0.02^{\circ} 2 \theta$ and a scan step time of $1.20 \mathrm{~s}$. To estimate quantities, we measured the diagnostic reflection areas, considering the full width at half maximum (FWHM) of the main minerals and then weighted by empirical factors or calculated parameters

Table 1

Number of samples analysed for the Porto dos Cacos and Quinta do Rouxinol workshops according to their typology.

\begin{tabular}{|c|c|c|c|c|c|c|c|}
\hline & Class $22=$ Almagro 50 & Class $23=$ Almagro 51c & Lusitana 9 & Class $20 / 21=$ Dressel 14 & Lusitana 3 & [A51c/L3]? & Total \\
\hline Quinta do Rouxinol & 49 & 49 & $32^{\mathrm{a}}$ & - & - & - & 130 \\
\hline Porto dos Cacos & 3 & 11 & - & 38 & $56^{\mathrm{b}}$ & 22 & 130 \\
\hline
\end{tabular}

a Six of these Lusitana 9 samples are sub-samples from the same amphora sherd.

b Among these Lusitana 3 samples, eight samples bear potters' marks (RVSTICI, TMM, CLARIAMI, AIVNIT (or TINVIA retro), three Germanus (CERF (Germanus), GERMAN (retro) and GERMA(ni)) and one non-identified. 
Table 2

Elemental compositions of Roman amphorae from the Porto dos Cacos workshop ( $\mu \mathrm{g} / \mathrm{g}$ unless specified otherwise).

\begin{tabular}{|c|c|c|c|c|c|c|c|c|c|c|c|c|c|c|c|c|c|c|c|c|c|c|c|c|}
\hline Sample & Type & $\mathrm{Na}_{2} \mathrm{O} \%$ & $\mathrm{~K}_{2} \mathrm{O} \%$ & $\mathrm{Fe}_{2} \mathrm{O}_{3} \mathrm{~T} \%$ & Sc & $\mathrm{Cr}$ & Co & $\mathrm{Zn}$ & As & $\mathrm{Rb}$ & $\mathrm{Sb}$ & Cs & $\mathrm{Ba}$ & La & $\mathrm{Ce}$ & $\mathrm{Nd}$ & $\mathrm{Sm}$ & $\mathrm{Eu}$ & $\mathrm{Tb}$ & $\mathrm{Yb}$ & Lu & $\mathrm{Hf}$ & Ta & Th \\
\hline PC-1 & L3 & 0.68 & 3.44 & 3.85 & 13.4 & 65.2 & 7.53 & 78.0 & 5.10 & 184 & 0.58 & 12.6 & 504 & 41.1 & 81.3 & 36.3 & 6.97 & 1.26 & 0.95 & 3.08 & 0.43 & 6.49 & 1.66 & 14.2 \\
\hline PC-3 & L3 & .77 & 3.72 & 4.68 & 15.3 & 71.2 & 9.44 & 84.7 & 6.13 & 196 & 0.52 & 15.1 & 572 & 47.7 & 95.7 & 41.8 & 8.38 & 1.37 & 1.04 & 3.48 & 0.46 & 7.01 & 1.88 & 18.0 \\
\hline PC-5 & L3 & 0.44 & 3.29 & 4.58 & 13.1 & 63.4 & 14.5 & 88.2 & 14.5 & 181 & 0.43 & 13.2 & 542 & 53.7 & 91.0 & 50.5 & 10.9 & 2.02 & 1.52 & 3.97 & 0.57 & 6.84 & 1.81 & 17.0 \\
\hline PC-9 & L3 & 0.43 & 3.28 & 4.62 & 12.7 & 60.4 & 16.1 & 83.7 & 19.3 & 184 & 0.56 & 13.4 & 536 & 53.5 & 84.6 & 49.8 & 10.3 & 1.87 & 1.38 & 3.78 & 0.55 & 6.69 & 1.85 & 16.4 \\
\hline PC-12 & L3 & 0.41 & 3.28 & 4.27 & 11.3 & 54.9 & 11.9 & 75.5 & 16.7 & 169 & 0.56 & 11.6 & 484 & 49.2 & 78.9 & 45.8 & 9.74 & 1.62 & 1.19 & 3.79 & 0.45 & 7.12 & 1.88 & 6.90 \\
\hline PC-14 & L3 & 0.71 & 3.46 & 5.01 & 12.6 & 59.9 & 8.69 & 90.6 & 6.70 & 176 & 0.53 & 12.3 & 456 & 41.1 & 76.7 & 36.2 & 7.17 & 1.13 & 0.81 & 3.26 & 0.49 & 6.75 & 1.77 & 16.2 \\
\hline PC-15 & L3 & 0.74 & 3.37 & 4.43 & 12.0 & 53.4 & 10.2 & 76.9 & 4.63 & 170 & 0.74 & 11.0 & 433 & 50.8 & 99.8 & 45.2 & 8.65 & 1.33 & 1.05 & 3.65 & 0.55 & 8.19 & 1.78 & 8.60 \\
\hline PC-16 & L3 & 0.42 & 3.33 & 4.55 & 12.6 & 61.3 & 23.3 & 87.9 & 19.1 & 172 & 0.57 & 13.4 & 514 & 55.2 & 87.1 & 0.70 & 10.4 & 1.95 & 1.33 & 3.68 & 0.63 & 6.63 & 1.75 & 16.4 \\
\hline PC-20 & L3 & 0.51 & 3.25 & 4.96 & 12.9 & 71.0 & 12.7 & 78.4 & 16.6 & 180 & 0.51 & 13.5 & 516 & 47.3 & 77.8 & 42.5 & 8.65 & 1.51 & 1.15 & 3.78 & 0.53 & 7.27 & 1.87 & 17.6 \\
\hline PC-21 & L3 & 0.55 & 3.28 & 4.47 & 12.8 & 66.8 & 10.5 & 67.1 & 17.9 & 184 & 0.47 & 13.4 & 476 & 42.3 & 84.6 & 40.5 & 8.22 & 1.45 & 1.20 & 3.29 & 0.53 & 6.69 & 1.89 & 17.1 \\
\hline PC-22 & L3 & .38 & 3.20 & 4.69 & 14.3 & 68.7 & 17.8 & 92.1 & 26.7 & 189 & 0.55 & 15.4 & 676 & 32.7 & 55.6 & 34.1 & 7.09 & 1.38 & 1.02 & 3.23 & 0.53 & 5.79 & 1.92 & 15.5 \\
\hline PC-23 & L3 & 0.50 & 3.30 & 4.33 & 12.6 & 64.9 & 12.1 & 60.2 & 19.0 & 189 & 0.53 & 13.2 & 622 & 46.4 & 73.0 & 42.5 & 8.79 & 1.61 & 1.24 & 3.63 & 0.54 & 7.02 & 1.94 & 17.2 \\
\hline PC-24 & L3 & 0.51 & 3.25 & 4.47 & 12.8 & 72.7 & 12.9 & 67.0 & 20.7 & 185 & 0.49 & 12.9 & 572 & 47.2 & 76.9 & 43.4 & 9.13 & 1.69 & 1.23 & 3.70 & 0.56 & 7.40 & 1.76 & 17.1 \\
\hline PC-26 & L3 & .43 & 3.34 & 4.43 & 12.7 & 61.0 & 13.6 & 74.3 & 20.2 & 186 & 0.58 & 13.1 & 490 & 48.9 & 75.0 & 46.30 & 9.36 & 1.70 & 1.32 & 3.67 & 0.54 & 7.19 & 1.79 & 17.4 \\
\hline PC-29 & L3 & 0.39 & 3.20 & 3.99 & 12.1 & 57.7 & 11.7 & 68.9 & 16.1 & 159 & 0.45 & 11.3 & 572 & 39.9 & 57.4 & 34.2 & 8.20 & 1.36 & 1.01 & 2.85 & 0.51 & 5.63 & 1.54 & 14.5 \\
\hline PC-31 & L3 & 0.80 & 3.40 & 5.02 & 12.7 & 56.0 & 8.74 & 87.9 & 5.86 & 187 & 0.67 & 12.0 & 493 & 41.1 & 78.1 & 36.9 & 7.45 & 1.23 & 0.97 & 2.75 & 0.50 & 7.82 & 1.79 & 17.2 \\
\hline PC-32 & L3 & .42 & 3.24 & 4.53 & 12.6 & 66.3 & 19.7 & 78.7 & 20.0 & 182 & 0.53 & 12.9 & 560 & 50.1 & 75.9 & 48.1 & 9.98 & 1.93 & 1.34 & 3.31 & 0.61 & 6.69 & 1.88 & 16.0 \\
\hline PC-34 & L3 & 0.51 & 3.06 & 5.86 & 13.2 & 60.2 & 6.97 & 72.3 & 10.6 & 199 & 0.78 & 14.1 & 467 & 68.0 & 84.6 & 47.3 & 8.61 & 1.26 & 0.97 & 3.22 & 0.52 & 8.52 & 1.84 & 19.8 \\
\hline PC-37 & L3 & 0.53 & 3.36 & 4.95 & 14.1 & 86.1 & 17.7 & 86.1 & 22.2 & 197 & 0.43 & 15.3 & 577 & 49.8 & 93.3 & 48.6 & 10.6 & 2.01 & 1.41 & 3.44 & 0.68 & 6.61 & 1.88 & 16.4 \\
\hline PC-38 & L3 & .73 & 3.16 & 4.20 & 13.1 & 67.5 & 11.6 & 74.9 & 12.1 & 178 & 0.63 & 12.5 & 495 & 46.2 & 90.1 & 41.8 & 8.58 & 1.48 & 1.15 & 3.11 & 0.56 & 7.70 & 1.85 & 17.1 \\
\hline PC-39 & L3 & 0.62 & 3.25 & 4.52 & 13.7 & 65.6 & 12.4 & 75.6 & 18.2 & 183 & 0.49 & 14.0 & 498 & 43.2 & 86.0 & 41.8 & 8.90 & 1.62 & 1.17 & 3.21 & 0.53 & 6.94 & 1.89 & 16.4 \\
\hline PC-41 & L3 & 0.56 & 3.24 & 4.61 & 13.2 & 65.5 & 12.0 & 72.8 & 18.4 & 184 & 0.44 & 14.1 & 606 & 42.3 & 80.5 & 41.6 & 8.40 & 1.53 & 1.10 & 3.34 & 0.49 & 6.77 & 1.82 & 16.1 \\
\hline PC-47 & L3 & .54 & 3.38 & 3.38 & 10.8 & 55.5 & 10.0 & 48.5 & 13.9 & 137 & 0.39 & 9.31 & 593 & 29.9 & 53.5 & 21.9 & 5.50 & 0.77 & 0.57 & 2.05 & 0.33 & 5.30 & 1.49 & 13.3 \\
\hline PC-48 & L3 & 0.37 & 3.41 & 4.60 & 13.6 & 66.1 & 13.4 & 79.5 & 21.6 & 179 & 0.52 & 14.1 & 1030 & 34.1 & 55.9 & 29.2 & 5.95 & 1.05 & 0.90 & 3.06 & 0.42 & 6.39 & 1.98 & 16.7 \\
\hline PC-49 & L3 & 0.36 & 3.40 & 4.81 & 12.7 & 65.5 & 17.5 & 78.9 & 20.6 & 191 & 0.60 & 12.9 & 554 & 43.1 & 65.2 & 37.8 & 7.89 & 1.54 & 1.18 & 3.11 & 0.51 & 5.93 & 1.84 & 16.1 \\
\hline PC-50 & L3 & 0.79 & 3.62 & 4.68 & 15.2 & 75.6 & 9.57 & 84.2 & 5.86 & 197 & 0.67 & 14.6 & 534 & 48.6 & 90.6 & 40.6 & 8.41 & 1.33 & 1.01 & 3.17 & 0.52 & 6.99 & 1.99 & 17.9 \\
\hline PC-52 & L3 & 0.42 & 3.20 & 4.55 & 13.3 & 65.8 & 14.4 & 80.6 & 12.7 & 190 & 0.53 & 13.8 & 485 & 51.4 & 88.6 & 51.0 & 11.2 & 2.02 & 1.49 & 3.95 & 0.60 & 6.99 & 1.95 & 16.8 \\
\hline PC-53 & L3 & 0.33 & 3.18 & 4.98 & 14.0 & 72.1 & 16.4 & 85.8 & 25.0 & 191 & 0.36 & 13.8 & 540 & 55.5 & 83.0 & 52.8 & 11.5 & 2.11 & 1.56 & 4.24 & 0.66 & 7.30 & 2.09 & 18.3 \\
\hline PC-54 & L3 & 0.35 & 3.09 & 4.83 & 13.1 & 65.2 & 17.8 & 84.5 & 18.9 & 187 & 0.56 & 12.8 & 529 & 54.5 & 83.2 & 50.3 & 10.8 & 1.85 & 1.48 & 4.01 & 0.62 & 7.73 & 1.86 & 18.4 \\
\hline PC-56 & L3 & 0.63 & 3.13 & 3.74 & 12.3 & 58.1 & 6.53 & 60.6 & 9.16 & 169 & 0.44 & 11.4 & 573 & 33.6 & 68.0 & 31.0 & 6.22 & 0.99 & 0.85 & 2.64 & 0.42 & 6.57 & 1.51 & 15.0 \\
\hline PC-57 & L3 & 0.46 & 3.15 & 4.57 & 12.1 & 57.7 & 12.1 & 68.1 & 17.0 & 184 & 0.46 & 12.3 & 552 & 41.2 & 71.8 & 37.2 & 7.88 & 1.26 & 1.04 & 3.25 & 0.50 & 7.55 & 1.69 & 17.5 \\
\hline PC-58 & L3 & 0.82 & 3.26 & 4.25 & 11.6 & 55.0 & 11.7 & 62.5 & 6.29 & 183 & 0.67 & 12.0 & 608 & 44.7 & 90.3 & 42.9 & 8.70 & 1.38 & 1.19 & 3.67 & 0.56 & 7.96 & 1.81 & 17.6 \\
\hline PC-59 & L3 & .42 & 3.36 & 4.63 & 13.2 & 63.6 & 16.3 & 81.1 & 21.4 & 201 & 0.50 & 14.1 & 485 & 48.8 & 75.2 & 47.2 & 10.0 & 1.83 & 1.41 & 3.96 & 0.62 & 7.03 & 1.89 & 17.2 \\
\hline PC-62 & L3 & 0.34 & 3.12 & 5.11 & 14.4 & 66.8 & 17.8 & 79.3 & 21.7 & 202 & 0.55 & 14.8 & 494 & 58.1 & 83.7 & 56.1 & 12.5 & 2.27 & 1.72 & 4.56 & 0.69 & 7.24 & 1.96 & 18.0 \\
\hline PC-63 & L3 & 0.38 & 3.15 & 4.47 & 13.3 & 63.5 & 16.4 & 77.0 & 18.6 & 196 & 0.52 & 13.7 & 510 & 48.2 & 76.5 & 50.9 & 11.1 & 2.00 & 1.61 & 4.06 & 0.62 & 6.87 & 1.82 & 17.1 \\
\hline PC-64 & L3 & .34 & 3.29 & 5.24 & 14.6 & 68.6 & 20.8 & 78.8 & 22.4 & 208 & 0.60 & 14.9 & 515 & 59.0 & 85.2 & 55.2 & 12.9 & 2.31 & 1.69 & 4.49 & 0.70 & 7.56 & 1.91 & 18.8 \\
\hline PC-65 & L3 & 0.34 & 3.30 & 5.10 & 14.4 & 71.2 & 17.9 & 78.3 & 22.7 & 209 & 0.51 & 14.7 & 522 & 59.5 & 91.3 & 57.9 & 11.9 & 2.26 & 1.66 & 4.76 & 0.68 & 7.23 & 1.96 & 18.4 \\
\hline PC-66 & L3 & .41 & 3.38 & 5.05 & 13.7 & 70.6 & 20.2 & 77.9 & 21.1 & 220 & 0.65 & 14.8 & 512 & 53.8 & 118 & 50.7 & 10.5 & 2.04 & 1.42 & 4.22 & 0.63 & 7.03 & 1.80 & 16.8 \\
\hline PC-67 & L3 & 39 & 3.54 & 5.10 & 14.2 & 68.9 & 15.3 & 74.4 & 22.5 & 209 & 0.60 & 14.8 & 488 & 58.4 & 86.8 & 55.8 & 11.4 & 2.11 & 1.62 & 4.40 & 0.69 & 7.41 & 2.06 & 18.6 \\
\hline PC-68 & L3 & .43 & 3.10 & 4.41 & 12.4 & 59.0 & 16.8 & 70.5 & 15.6 & 187 & 0.49 & 12.7 & 492 & 49.7 & 90.9 & 46.5 & 10.2 & 1.73 & 1.32 & 3.41 & 0.55 & 7.52 & 1.77 & 17.6 \\
\hline PC-69 & L3 & 0.50 & 3.33 & 4.74 & 13.3 & 62.4 & 14.7 & 76.9 & 17.8 & 196 & 0.59 & 13.9 & 455 & 51.1 & 82.0 & 45.4 & 10.2 & 1.76 & 1.31 & 3.84 & 0.57 & 7.17 & 1.78 & 17.0 \\
\hline PC-70 & L3 & .25 & 2.10 & 4.91 & 13.9 & 65.7 & 15.4 & 80.5 & 14.5 & 199 & 0.65 & 14.5 & 516 & 37.7 & 84.8 & 53.4 & 7.23 & 2.09 & 1.53 & 4.27 & 0.64 & 7.55 & 2.19 & 18.6 \\
\hline PC-118 & L3 & 0.42 & 3.25 & 4.89 & 13.5 & 66.0 & 15.9 & 81.9 & 19.1 & 183 & 0.56 & 13.4 & 399 & 53.1 & 97.1 & 52.7 & 10.5 & 1.85 & 1.39 & 4.10 & 0.59 & 7.83 & 2.03 & 18.4 \\
\hline PC-119 & L3 & .40 & 3.21 & 4.67 & 13.3 & 63.6 & 14.4 & 85.6 & 18.4 & 187 & 0.61 & 12.8 & 392 & 55.1 & 95.3 & 56.4 & 10.3 & 2.03 & 1.48 & 4.28 & 0.65 & 8.19 & 1.96 & 18.7 \\
\hline PC-120 & L3 & 67 & 3.29 & 4.50 & 13.9 & 67.3 & 9.24 & 78.1 & 7.60 & 176 & 0.54 & 12.4 & 465 & 50.0 & 94.4 & 44.2 & 7.74 & 1.31 & 1.05 & 3.28 & 0.51 & 7.71 & 1.79 & 17.8 \\
\hline PC-121 & L3 & 0.43 & 3.17 & 4.65 & 12.9 & 61.3 & 14.2 & 73.2 & 16.8 & 180 & 0.49 & 12.5 & 409 & 52.0 & 84.2 & 49.0 & 8.88 & 1.75 & 1.32 & 4.05 & 0.59 & 8.54 & 1.86 & 18.7 \\
\hline PC-122 & L3 & 0.45 & 3.36 & 4.73 & 12.8 & 60.7 & 15.0 & 78.9 & 15.4 & 183 & 0.53 & 12.8 & 442 & 54.9 & 94.2 & 52.7 & 10.1 & 1.78 & 1.31 & 4.10 & 0.60 & 8.55 & 1.81 & 19.7 \\
\hline PC-123 & L3 & .45 & 3.41 & 4.87 & 14.2 & 65.2 & 17.1 & 187 & 20.7 & 202 & 0.53 & 14.7 & 471 & 52.8 & 76.4 & 46.7 & 10.4 & 1.96 & 1.52 & 4.53 & 0.65 & 7.44 & 2.07 & 17.9 \\
\hline PC-124 & L3 & .47 & 3.31 & 4.86 & 13.8 & 64.0 & 16.1 & 84.2 & 15.5 & 197 & 0.49 & 14.2 & 414 & 50.3 & 76.5 & 50.1 & 10.0 & 1.95 & 1.46 & 4.16 & 0.60 & 6.75 & 1.78 & 16.0 \\
\hline PC-125 & L3 & .48 & 3.13 & 4.77 & 12.7 & 59.6 & 10.2 & 60.2 & 20.8 & 184 & 0.56 & 13.4 & 447 & 44.9 & 77.8 & 42.4 & 8.52 & 1.35 & 1.08 & 3.61 & 0.52 & 8.05 & 1.84 & 18.3 \\
\hline PC-126 & L3 & .53 & 3.41 & 4.74 & 13.3 & 64.9 & 15.3 & 85.5 & 15.5 & 186 & 0.48 & 13.3 & 402 & 47.4 & 72.5 & 45.8 & 9.38 & 1.75 & 1.28 & 3.77 & 0.61 & 6.99 & 1.82 & 16.2 \\
\hline PC-127 & L3 & 80 & 3.46 & 3.92 & 11.6 & 54.5 & 9.43 & 74.7 & 4.29 & 171 & 0.60 & 10.7 & 496 & 41.6 & 82.0 & 39.9 & 7.73 & 1.24 & 1.02 & 3.27 & 0.48 & 6.90 & 1.70 & 16.4 \\
\hline PC-128 & L3 & 38 & 3.26 & 5.23 & 12.6 & 67.6 & 16.2 & 82.5 & 34.3 & 188 & 0.59 & 12.6 & 589 & 43.6 & 74.6 & 42.7 & 8.63 & 1.73 & 1.23 & 3.64 & 0.53 & 5.14 & 1.78 & 15.3 \\
\hline PC-129 & L3 & 37 & 3.03 & 4.67 & 12.9 & 60.3 & 12.5 & 78.3 & 16.4 & 181 & 0.48 & 12.7 & 421 & 55.2 & 92.5 & 52.4 & 10.5 & 1.82 & 1.41 & 4.30 & 0.65 & 8.18 & 1.72 & 18.70 \\
\hline PC -130 & L3 & .79 & 3.41 & 4.04 & 11.8 & 53.5 & 13.6 & 73.8 & 4.45 & 180 & 0.62 & 11.5 & 438 & 46.0 & 84.3 & 40.3 & 7.76 & 1.28 & 1.04 & 3.39 & 0.56 & 7.73 & 1.81 & 16.5 \\
\hline PC-131 & L3 & 0.45 & 3.15 & 4.29 & 11.7 & 58.1 & 13.8 & 70.8 & 17.8 & 174 & 0.42 & 11.7 & 542 & 41.6 & 63.4 & 35.6 & 7.50 & 1.30 & 0.96 & 3.23 & 0.46 & 6.80 & 1.69 & 15.8 \\
\hline PC-11 & & & 3.08 & 4.10 & & 59.4 & 1.03 & & 7.38 & 178 & & & & & & & & 1.08 & 0.96 & 3.1 & 0.50 & & 1.76 & \\
\hline PC-17 & A51c & .59 & 3.01 & 4.33 & 10.7 & 51.5 & 54.4 & 95.2 & 10.4 & 156 & 0.62 & 10.5 & 517 & 40.6 & 75.6 & 34.1 & 6.65 & 0.88 & 0.83 & 2.67 & 0.43 & 8.30 & 1.61 & 19.4 \\
\hline PC-19 & A51c & 01 & 5.21 & 4.80 & 11.5 & 51.0 & 8.92 & 80.1 & 14.2 & 167 & 0.53 & 10.9 & 565 & 59.7 & 68.8 & 31.3 & 10.7 & 1.01 & 0.87 & 2.61 & 0.42 & 6.69 & 1.59 & 16.1 \\
\hline PC-28 & A51c & 65 & 3.28 & 5.16 & 13.0 & 57.2 & 10.1 & 83.1 & 8.04 & 190 & 0.69 & 12.6 & 437 & 42.8 & 82.0 & 39.8 & 7.75 & 1.29 & 1.06 & 3.26 & 0.50 & 6.93 & 1.77 & 16.9 \\
\hline PC-44 & A51c & 66 & 3.52 & 5.21 & 13.0 & 59.0 & 9.19 & 76.3 & 8.19 & 188 & 0.58 & 12.7 & 518 & 43.1 & 79.4 & 37.1 & 7.19 & 1.16 & 0.93 & 2.99 & 0.49 & 7.06 & 1.73 & 17.5 \\
\hline PC-112 & A51c & .78 & 3.29 & 4.95 & 14.6 & 66.7 & 11.9 & 79.5 & 13.4 & 190 & 0.75 & 14.3 & 441 & 48.1 & 93.3 & 45.6 & 9.16 & 1.50 & 1.24 & 3.59 & 0.54 & 7.28 & 1.97 & 18.0 \\
\hline PC-113 & A51c & 62 & 2.87 & 4.91 & 13.6 & 62.7 & 8.13 & 63.0 & 9.92 & 178 & 0.66 & 13.7 & 361 & 52.1 & 100 & 47.1 & 9.10 & 1.50 & 1.13 & 3.34 & 0.49 & 7.23 & 1.73 & 17.3 \\
\hline PC-114 & A51c & .64 & 3.37 & 5.02 & 12.0 & 52.4 & 9.14 & 77.9 & 9.25 & 188 & 0.69 & 11.7 & 378 & 45.4 & 91.0 & 43.1 & 8.26 & 1.17 & 1.06 & 3.38 & 0.52 & 8.36 & 1.70 & 20.0 \\
\hline PC-115 & A51c & 0.78 & 3.39 & 5.32 & 13.5 & 58.5 & 8.96 & 79.0 & 7.07 & 190 & 0.60 & 12.9 & 401 & 47.4 & 90.8 & 41.4 & 8.38 & 1.26 & 1.05 & 3.45 & 0.53 & 8.25 & 1.92 & 18.6 \\
\hline PC-116 & A51c & 0.68 & 3.38 & 4.23 & 13.8 & 63.2 & 8.60 & 82.4 & 6.79 & 178 & 0.45 & 12.6 & 45 & 47.4 & 91.7 & 40.7 & 7.86 & 1.31 & 1.02 & 3.34 & 0.53 & 6.72 & 1.65 & 16.4 \\
\hline PC-117 & A51c & 0.75 & 3.24 & 5.52 & 13.8 & 59.6 & 10.7 & 93.4 & 8.50 & 184 & 0.65 & 13.1 & 392 & 47.5 & 92.6 & 42.7 & 8.44 & 1.30 & 1.10 & 3.56 & 0.57 & 7.16 & 2.27 & 18.0 \\
\hline PC-74 & D14 & 68 & 3.13 & 4.96 & 5.6 & 75.7 & 9.07 & 76.0 & 19.8 & 200 & 0.63 & 16.4 & 542 & 43.5 & & 39.10 & 7.89 & 1.37 & 1.11 & 3.18 & 0.47 & 6.62 & 2.10 & 17.7 \\
\hline PC-75 & D14 & .55 & 3.03 & 4.62 & 12.6 & 67.5 & 6.69 & 63.0 & 22.0 & 172 & 0.61 & 13.3 & 621 & 40.7 & 81.9 & 36.8 & 7.50 & 1.18 & 0.97 & 3.27 & 0.46 & 7.90 & 1.89 & 18.3 \\
\hline PC-76 & D14 & 0.57 & 3.16 & 4.97 & 13.7 & 66.3 & 7.66 & 71.0 & 25.0 & 182 & 0.56 & 14.6 & 608 & 44.3 & 85.4 & 40.0 & 7.97 & 1.26 & 0.99 & 3.33 & 0.51 & 8.37 & 1.96 & 19.4 \\
\hline PC-77 & D14 & 0.49 & 3.06 & 4.60 & 13.5 & 60.5 & 7.99 & 63.9 & 13.5 & 189 & 0.55 & 14.1 & 466 & 43.0 & 83.9 & 40.3 & 8.33 & 1.30 & 1.08 & 3.14 & 0.50 & 7.32 & 1.80 & 17.7 \\
\hline PC-78 & D14 & 0.56 & 2.84 & 4.79 & 13.9 & 65.5 & 7.03 & 65.1 & 16.1 & 183 & 0.61 & 14.0 & 569 & 38.9 & 79.3 & 37.3 & 8.03 & 1.21 & 0.97 & 3.19 & 0.49 & 7.24 & 1.86 & 18.6 \\
\hline PC-79 & D14 & 0.50 & 2.78 & 4.26 & 12.1 & 58.1 & 6.88 & 65.1 & 13.1 & 162 & n.d. & 12.3 & 383 & 44.8 & 82.8 & 39.7 & 7.78 & 1.20 & 0.90 & 3.00 & 0.48 & 7.54 & 1.59 & 17.5 \\
\hline
\end{tabular}


Table 2 (continued)

\begin{tabular}{|c|c|c|c|c|c|c|c|c|c|c|c|c|c|c|c|c|c|c|c|c|c|c|c|c|c|}
\hline Sample & Type & $\mathrm{Na}_{2} \mathrm{O} \%$ & $\mathrm{~K}_{2} \mathrm{O} \%$ & $\mathrm{Fe}_{2} \mathrm{O}_{3} \mathrm{~T} \%$ & Sc & $\mathrm{Cr}$ & Co & $\mathrm{Zn}$ & As & $\mathrm{Rb}$ & $\mathrm{Sb}$ & Cs & $\mathrm{Ba}$ & $\mathrm{La}$ & $\mathrm{Ce}$ & $\mathrm{Nd}$ & $\mathrm{Sm}$ & $\mathrm{Eu}$ & $\mathrm{Tb}$ & $\mathrm{Yb}$ & Lu & Hf & Ta & Th & $\mathrm{U}$ \\
\hline PC-80 & D14 & 0.51 & 3.19 & 4.82 & 13.6 & 63.0 & 8.35 & 62.9 & 18.3 & 187 & 0.55 & 13.8 & 465 & 40.0 & 77.5 & 35.3 & 7.14 & 1.14 & 0.89 & 2.93 & 0.44 & 6.91 & 1.89 & 17.7 & 5.81 \\
\hline PC-81 & D14 & 0.54 & 3.09 & 4.79 & 13.2 & 63.2 & 7.17 & 67.1 & 21.5 & 191 & 0.53 & 13.6 & 650 & 31.6 & 63.9 & 31.1 & 6.39 & 1.06 & 0.84 & 2.88 & 0.46 & 6.65 & 1.76 & 16.7 & 5.75 \\
\hline PC-82 & D14 & 0.59 & 3.35 & 4.55 & 13.1 & 64.4 & 7.79 & 59.4 & 17.2 & 189 & 0.52 & 13.7 & 481 & 35.4 & 68.9 & 34.1 & 6.93 & 1.20 & 0.91 & 2.82 & 0.41 & 5.87 & 1.79 & 15.1 & 6.41 \\
\hline PC-83 & D14 & 0.71 & 3.65 & 4.88 & 13.9 & 69.0 & 12.1 & 75.4 & 12.8 & 208 & 0.73 & 13.6 & 523 & 41.9 & 90.8 & 42.0 & 8.38 & 1.44 & 1.17 & 3.66 & 0.54 & 6.32 & 1.99 & 17.5 & 4.39 \\
\hline PC-84 & D14 & 0.49 & 3.33 & 4.86 & 13.9 & 72.8 & 9.80 & 67.5 & 28.2 & 203 & 0.61 & 14.8 & 541 & 36.7 & 72.5 & 35.7 & 7.22 & 1.30 & 0.98 & 3.13 & 0.47 & 5.89 & 1.88 & 15.8 & 6.96 \\
\hline PC-85 & D14 & 0.59 & 3.33 & 5.05 & 15.0 & 73.9 & 8.63 & 67.5 & 23.8 & 200 & 0.57 & 15.3 & 530 & 36.8 & 72.4 & 35.3 & 7.25 & 1.31 & 0.88 & 3.23 & 0.47 & 6.63 & 2.00 & 16.7 & 6.99 \\
\hline PC-86 & D14 & 0.62 & 3.27 & 4.48 & 13.1 & 63.3 & 11.5 & 129 & 19.8 & 167 & 0.53 & 11.4 & 935 & 33.0 & 71.4 & 32.0 & 6.61 & 1.06 & 0.72 & 3.39 & 0.46 & 5.51 & 1.70 & 15.2 & 4.73 \\
\hline PC-87 & D14 & 0.67 & 3.45 & 4.81 & 13.7 & 67.8 & 9.75 & 73.8 & 15.7 & 202 & 0.66 & 13.3 & 520 & 39.2 & 79.9 & 38.8 & 7.87 & 1.36 & 1.01 & 3.05 & 0.52 & 6.18 & 1.79 & 16.8 & 6.22 \\
\hline PC-88 & D14 & 0.61 & 3.35 & 4.37 & 12.8 & 58.9 & 9.67 & 76.6 & 19.6 & 172 & 0.61 & 13.5 & 525 & 38.8 & 74.7 & 37.3 & 7.77 & 1.39 & 0.98 & 3.14 & 0.42 & 6.03 & 1.77 & 16.3 & 6.73 \\
\hline PC-89 & D14 & 0.60 & 3.48 & 4.83 & 13.3 & 65.0 & 9.43 & 69.8 & 22.0 & 195 & 0.62 & 12.9 & 589 & 40.5 & 79.1 & 38.7 & 7.96 & 1.40 & 0.97 & 3.20 & 0.56 & 6.30 & 1.86 & 17.2 & 6.20 \\
\hline PC-90 & D14 & 0.53 & 3.50 & 4.70 & 13.6 & 67.7 & 9.21 & 66.6 & 21.4 & 199 & 0.57 & 13.9 & 626 & 35.4 & 69.2 & 33.4 & 6.79 & 1.22 & 0.94 & 3.14 & 0.58 & 6.01 & 1.89 & 16.2 & 6.64 \\
\hline PC-91 & D14 & 0.73 & 3.49 & 3.98 & 11.6 & 56.1 & 8.12 & 59.3 & 14.3 & 160 & 0.58 & 11.2 & 454 & 40.1 & 75.5 & 36.4 & 7.65 & 1.16 & 0.99 & 3.10 & 0.48 & 6.75 & 1.84 & 15.2 & 4.88 \\
\hline PC-92 & D14 & 0.62 & 3.59 & 4.16 & 12.1 & 56.3 & 7.83 & 58.4 & 15.9 & 160 & 0.48 & 11.9 & 552 & 32.3 & 63.0 & 29.4 & 6.00 & 1.06 & 0.86 & 2.89 & 0.41 & 5.66 & 1.74 & 13.8 & 4.24 \\
\hline PC-93 & D14 & 0.61 & 3.41 & 4.78 & 13.9 & 66.0 & 7.32 & 64.9 & 15.9 & 182 & 0.63 & 14.0 & 505 & 47.0 & 81.3 & 38.1 & 8.01 & 1.22 & 0.93 & 3.13 & 0.50 & 7.74 & 2.05 & 17.9 & 5.79 \\
\hline PC-94 & D14 & 0.50 & 2.99 & 4.26 & 11.5 & 53.7 & 7.15 & 60.1 & 14.9 & 154 & 0.45 & 11.8 & 411 & 49.5 & 86.5 & 40.5 & 8.14 & 1.19 & 0.97 & 2.92 & 0.48 & 7.59 & 1.68 & 17.7 & 5.66 \\
\hline PC-95 & D14 & 0.65 & 3.10 & 5.89 & 12.3 & 62.1 & 8.22 & 62.7 & 23.9 & 164 & 0.63 & 12.3 & 612 & 33.8 & 64.8 & 29.4 & 6.24 & 0.94 & 0.86 & 2.81 & 0.43 & 6.55 & 1.89 & 16.0 & 6.48 \\
\hline PC-96 & D14 & 0.57 & 3.09 & 4.46 & 12.4 & 60.3 & 7.84 & 65.3 & 17.1 & 167 & 0.58 & 13.0 & 596 & 44.0 & 80.6 & 39.4 & 8.17 & 1.23 & 0.97 & 3.05 & 0.48 & 7.19 & 1.80 & 17.1 & 5.43 \\
\hline PC-97 & D14 & 0.59 & 3.37 & 4.55 & 12.9 & 59.0 & 7.53 & 64.8 & 17.5 & 184 & 0.42 & 13.8 & 454 & 48.8 & 87.5 & 40.9 & 8.15 & 1.23 & 0.97 & 3.24 & 0.49 & 7.90 & 1.79 & 18.6 & 6.30 \\
\hline PC-98 & D14 & 0.56 & 3.18 & 4.71 & 13.5 & 67.5 & 14.2 & 77.5 & 18.1 & 186 & 0.52 & 13.70 & 501 & 48.0 & 95.3 & 48.0 & 10.4 & 1.79 & 1.29 & 4.00 & 0.57 & 7.07 & 1.94 & 17.9 & 6.88 \\
\hline PC-99 & D14 & 52 & 3.25 & 5.37 & 14.5 & 67.2 & 9.78 & 75.9 & 20.8 & 201 & 0.53 & 15.4 & 506 & 48.5 & 93.3 & 44.1 & 8.72 & 1.43 & 1.14 & 3.24 & 0.49 & 7.40 & 2.03 & 18.8 & 6.37 \\
\hline PC -100 & D14 & 67 & 3.97 & 4.91 & 14.9 & 75.8 & 15.2 & 83.5 & 22.8 & 194 & 0.59 & 15.3 & 510 & 57.1 & 99.0 & 52.4 & 12.1 & 2.11 & 1.56 & 4.35 & 0.65 & 6.87 & 1.98 & 16.9 & 7.44 \\
\hline PC-101 & D14 & 0.71 & 3.49 & 4.53 & 13.3 & 69.4 & 15.8 & 77.7 & 21.3 & 188 & 0.47 & 13.8 & 523 & 54.1 & 79.7 & 46.0 & 10.5 & 1.89 & 1.26 & 3.79 & 0.61 & 6.31 & 1.92 & 15.7 & 6.43 \\
\hline PC-102 & D14 & 0.68 & 3.72 & 4.28 & 12.8 & 60.2 & 16.6 & 72.5 & 20.8 & 182 & 0.47 & 13.3 & 473 & 51.2 & 85.5 & 43.7 & 9.70 & 1.70 & 1.23 & 3.50 & 0.53 & 6.01 & 1.81 & 14.7 & 7.01 \\
\hline-103 & D14 & 0.63 & 3.87 & 4.78 & 14.0 & 72.7 & 8.29 & 70.9 & 20.2 & 183 & 0.59 & 14.1 & 525 & 41.0 & 70.0 & 33.4 & 7.60 & 1.30 & 1.04 & 3.24 & 0.52 & 5.68 & 1.88 & 15.3 & 6.73 \\
\hline PC-104 & D14 & 0.47 & 2.93 & 4.01 & 11.6 & 52.8 & 6.92 & 55.1 & 18.5 & 160 & 0.44 & 12.6 & 416 & 30.4 & 71.5 & 34.0 & 7.26 & 1.15 & 0.86 & 2.65 & 0.47 & 6.27 & 1.66 & 14.7 & 5.54 \\
\hline PC-105 & D14 & 0.61 & 3.51 & 4.61 & 13.2 & 61.0 & 6.44 & 63.0 & 24.7 & 165 & 0.51 & 13.3 & 564 & 36.7 & 61.4 & 30.6 & 6.56 & 1.06 & 0.85 & 2.64 & 0.42 & 6.03 & 1.78 & 15.0 & 4.99 \\
\hline PC-106 & D14 & 0.65 & 3.41 & 4.89 & 14.6 & 69.0 & 13.6 & 81.0 & 18.3 & 198 & 0.58 & 14.8 & 567 & 50.4 & 90.7 & 41.1 & 11.2 & 1.96 & 1.43 & 3.37 & 0.62 & 7.38 & 2.16 & 18.80 & 6.66 \\
\hline PC-107 & D14 & 0.58 & 3.26 & 5.11 & 14.5 & 69.7 & 9.96 & 70.1 & 19.0 & 197 & 0.51 & 15.5 & 469 & 46.2 & 94.6 & 42.2 & 8.75 & 1.42 & 1.13 & 3.28 & 0.53 & 7.64 & 1.90 & 18.7 & 6.72 \\
\hline PC -108 & D14 & 0.60 & 3.23 & 5.07 & 13.9 & 64.1 & 7.39 & 62.0 & 22.3 & 191 & 0.41 & 14.7 & 580 & 41.4 & 82.9 & 38.7 & 7.64 & 1.25 & 1.01 & 3.21 & 0.41 & 7.30 & 1.87 & 18.8 & 5.84 \\
\hline PC-109 & D14 & 0.52 & 3.02 & 4.69 & 13.5 & 66.0 & 6.60 & 60.6 & 26.4 & 181 & 0.55 & 14.0 & 618 & 40.2 & 77.0 & 36.5 & .52 & 1.25 & 0.97 & 3.14 & 0.49 & 7.79 & 1.99 & 19.4 & 6.25 \\
\hline & D14 & 0.74 & 3.84 & 4.73 & 14.5 & 72.2 & 8.40 & 72.4 & 18.4 & 187 & 0.50 & 14.9 & 45 & 46.2 & 77.0 & 36.0 & & 1.35 & 1.05 & 3.0 & 0.51 & 6.30 & 1.88 & 16.3 & 5.89 \\
\hline & D14 & 0.59 & 3.28 & 5.45 & 15.7 & 78.3 & 10.5 & 85.4 & & 203 & & & & 47.0 & 95.0 & & & 1.70 & & & 0.57 & & 2.12 & 19.6 & \\
\hline & & & & & & & & & & & & & & & & & & & & & & & & & \\
\hline & A50 & 0.66 & 3.13 & 4.80 & 14.1 & 69.7 & 11.3 & 73.7 & 16.8 & 183 & 0.76 & 13.2 & 468 & 51.2 & 94.7 & 45.9 & 9.23 & 1.50 & 1.19 & 3.47 & 0.58 & 7.82 & 1.94 & 18.7 & 4.43 \\
\hline PC-73 & A50 & 0.57 & 3.12 & 4.54 & 12.7 & 60.9 & & 67.3 & 9.81 & & 0.71 & & & 50.6 & 87.8 & 42.20 & 7.90 & 1.24 & & & 0.48 & 8.09 & 1.90 & 17.7 & 4.34 \\
\hline PC-2 & & & 2.81 & 4.07 & & 50.0 & & 52.8 & 9.41 & 164 & & 10.8 & & 48.2 & & & 7.90 & 1.03 & & 3.23 & 0.50 & 10.5 & 1.93 & 22.6 & 5.29 \\
\hline PC-4 & A51c/l & 0.69 & 3.61 & 4.42 & 14.9 & 68.9 & 10.0 & 85.2 & 9.29 & 187 & 0.55 & 14.6 & 508 & 39.2 & 75.6 & 35.8 & 7.35 & 1.33 & 0.93 & 3.16 & 0.48 & 5.85 & 1.76 & 15.1 & 5.11 \\
\hline PC-6 & A51c/ & 0.57 & 3.10 & 4.64 & 12.3 & 57.2 & & 65.4 & 13.5 & 181 & 0.77 & 12.2 & 423 & 52.9 & 82.6 & 42.1 & 7.80 & 1.20 & 0.99 & 3.41 & 0.51 & 8.82 & 1.76 & 18.7 & \\
\hline PC-7 & A51c & 0.66 & 3.12 & 4.99 & 2.6 & 55.8 & & 78.2 & 7.44 & 185 & 0.69 & 12.4 & 45 & 43.6 & 83.7 & 39.0 & 7.34 & 1.18 & 0.93 & 3.15 & 0.49 & 7.53 & 1.71 & 17.9 & 4.46 \\
\hline PC-8 & A51c/L3? & 0.38 & 3.12 & 4.76 & 13.5 & 69.3 & 7.31 & 76.5 & 18.0 & 176 & 0.75 & 12.5 & 536 & 40.3 & 69.5 & 32.6 & 6.86 & 1.18 & 0.78 & 3.02 & 0.44 & 7.10 & 1.89 & 16.5 & 5.38 \\
\hline PC-10 & A51c/L3? & 0.72 & 3.34 & 4.99 & 12.3 & 58.8 & 8.95 & 80.4 & 6.23 & 182 & 0.65 & 12.7 & 471 & 37.8 & 74.8 & 35.2 & 7.01 & 1.13 & 0.93 & 3.19 & 0.47 & 7.43 & 1.87 & 16.6 & 4.03 \\
\hline PC-13 & A51c/L3? & 0.68 & 3.13 & 4.15 & 12.3 & 61.6 & & 67.7 & 11.5 & 173 & 0.73 & 11.6 & 467 & 42.9 & 79.1 & 38.6 & 7.28 & 1.16 & 0.92 & 3.29 & 0.51 & 7.78 & 1.77 & 18.2 & 4.08 \\
\hline PC-18 & A51c/L3? & 0.53 & 3.09 & 4.76 & 12.3 & 57.8 & 8.79 & 59.5 & 5.88 & 188 & 0.51 & 12.9 & 566 & 54.2 & 101 & 46.6 & 8.76 & 1.32 & 1.12 & 3.07 & 0.53 & 8.58 & 1.74 & 20.0 & 4.49 \\
\hline PC-25 & A51c/L3? & 0.60 & 3.07 & 4.83 & 13.7 & 71.2 & 9.71 & 71.0 & 11.6 & 182 & 0.68 & 12.3 & 465 & 49.3 & 96.2 & 48.2 & 9.07 & 1.53 & 1.18 & 3.59 & 0.55 & 7.80 & 1.95 & 18.9 & 4.37 \\
\hline & A51c/L3? & 0.68 & 3.32 & 4.97 & 13.0 & 65.0 & & 64.2 & 17.0 & 182 & 0.71 & 11.8 & & 38.9 & 72.3 & 36.7 & 7.30 & 1.25 & 0.95 & 3.02 & 0.49 & 6.86 & 1.83 & 16.9 & 4.85 \\
\hline PC-30 & A51c/L3? & 0.52 & 3.10 & 3.96 & 11.9 & 57.6 & 10.4 & 67.8 & 13.9 & 171 & 0.44 & 12.4 & 471 & 41.8 & 67.1 & 36.9 & 7.84 & 1.40 & 1.01 & 2.98 & 0.49 & 6.05 & 1.58 & 14.5 & 6.77 \\
\hline PC-33 & A51c/L3? & 0.65 & 2.94 & 4.64 & 13.3 & 64.4 & 6.65 & 58.4 & 12.5 & 162 & 0.65 & 12.4 & 648 & 40.6 & 78.5 & 37.1 & 7.39 & 1.18 & 1.98 & 3.16 & 0.49 & 7.93 & 1.80 & 18.1 & 4.98 \\
\hline PC-35 & A51c/L3? & 0.57 & 2.81 & 4.14 & 11.0 & 52.1 & 5.85 & 54.1 & 10.3 & 153 & 0.58 & 10.6 & 520 & 42.0 & 74.6 & 37.4 & 7.35 & 1.12 & 0.88 & 2.92 & 0.47 & 7.03 & 1.46 & 16.8 & 4.03 \\
\hline PC-36 & A51c/L3? & 0.57 & 3.20 & 4.93 & 14.4 & 67.8 & 10.5 & 73.5 & 10.5 & 199 & 0.78 & 14.1 & 521 & 58.7 & 112 & 55.4 & 11.1 & 1.86 & 1.37 & 3.74 & 0.62 & 7.84 & 1.91 & 18.6 & 5.15 \\
\hline PC-40 & A51c/L3? & 0.45 & 2.70 & 2.61 & 6.0 & 44.0 & 2.95 & 44.0 & 10.1 & 91.6 & 0.39 & 4.97 & 250 & 36.0 & 62.0 & 30.1 & 5.73 & 0.46 & 0.90 & 2.50 & 0.35 & 5.67 & 0.83 & 14.5 & 3.16 \\
\hline PC-43 & A51c/L3? & 0.78 & 3.34 & 4.23 & 13.9 & 70.8 & 8.65 & 78.0 & 5.73 & 192 & 0.60 & 13.4 & 476 & 44.3 & 85.8 & 38.6 & 7.80 & 1.29 & 1.01 & 3.23 & 0.52 & 7.17 & 1.88 & 17.1 & 6.25 \\
\hline PC- 45 & A51c/L3? & 0.49 & 3.59 & 3.79 & 10.7 & 55.3 & 14.8 & 69.8 & 17.4 & 168 & 0.50 & 10.1 & 557 & 41.3 & 85.5 & 37.2 & 7.22 & 1.22 & 1.01 & 3.10 & 0.47 & 7.14 & 1.78 & 17.1 & 5.36 \\
\hline PC-46 & A51c/L3? & 0.72 & 3.82 & 4.10 & 14.3 & 70.1 & 8.24 & 89.7 & 4.70 & 194 & 0.46 & 13.6 & 535 & 52.4 & 90.7 & 40.9 & 7.89 & 1.36 & 0.89 & 2.92 & 0.48 & 5.87 & 1.73 & 15.5 & 5.45 \\
\hline PC-51 & A51c/L3? & 0.66 & 3.19 & 5.17 & 12.5 & 55.3 & 8.88 & 84.7 & 8.26 & 190 & 0.72 & 12.6 & 415 & 40.9 & 78.5 & 37.0 & 8.10 & 1.24 & 0.95 & 2.96 & 0.48 & 6.42 & 1.75 & 16.0 & 4.19 \\
\hline PC-55 & A51c/L3? & 0.76 & 3.16 & 4.86 & 12.1 & 55.3 & 7.92 & 74.0 & 7.91 & 184 & 0.62 & 12.0 & 418 & 44.0 & 84.4 & 39.8 & 7.78 & 1.18 & 0.98 & 2.96 & 0.53 & 7.74 & 1.75 & 17.9 & 4.75 \\
\hline PC-60 & A51c/L3? & 0.63 & 3.31 & 4.65 & 14.7 & 70.4 & 11.6 & 64.3 & 11.7 & 179 & 0.49 & 14.7 & 700 & 26.6 & 55.0 & 24.0 & 4.94 & 0.76 & 0.71 & 2.44 & 0.39 & 5.81 & 1.67 & 15.6 & 4.48 \\
\hline PC-61 & A51c/L3? & 0.69 & 3.15 & 4.51 & 11.4 & 52.3 & 7.81 & 63.5 & 11.2 & 173 & 0.50 & 12.4 & 552 & 34.1 & 67.6 & 31.5 & 6.19 & 0.97 & 0.88 & 2.71 & 0.43 & 6.93 & 1.69 & 16.2 & 4.28 \\
\hline
\end{tabular}

(Schultz, 1964; Biscaye, 1965; Martín-Pozas, 1968). The diagnostic peaks used and the corresponding reflection powers (in parenthesis) were the following: quartz - $3.35 \AA$ (2), alkali feldspar $3.25 \AA$ ( 1 ), plagioclase $-3.20 \AA$ (1), phyllosilicates - $4.46(0.1)$, anatase - $3.52 \AA(1)$, hematite -2.70 (1.3) and mullite - $3.38 \AA$ (1). Given the uncertainties intrinsic to this semi-quantitative method, the results obtained should only be taken as rough estimates of mineral percentages.

For the evaluation of data, and considering that the compositional data generated by INAA is considerable, multivariate statistical methods were applied, using the Statistica program (StatSoft, 2008) and the JMP7 program (SAS). Hierarchical and non-hierarchical cluster analyses (joining-tree and k-means methods) were used to identify possible groups and outliers, after which different techniques were employed for group refinement and classification. The amalgamation rule employed in the joining-tree clustering was the unweighted pair-group average, also referred to as UPGMA (unweighted pair-group method using arithmetic averages), as well as Ward's method. In the present case study, the Euclidean mean, representing the measure of the actual geometric distance between objects in space, was used as the similarity coefficient to identify outliers. The Pearson correlation coefficient was used to evaluate the correlation between chemical parameters and samples. Principal component analysis 
Table 3

Elemental compositions of Roman amphorae from the Quinta do Rouxinol workshop ( $\mu \mathrm{g} / \mathrm{g}$ unless specified otherwise).

\begin{tabular}{|c|c|c|c|c|c|c|c|c|c|c|c|c|c|c|c|c|c|c|c|c|c|c|c|}
\hline Sample & Type & $\mathrm{Na}_{2} \mathrm{O} \%$ & $\mathrm{~K}_{2} \mathrm{O} \%$ & $\mathrm{Fe}_{2} \mathrm{O}_{3} \mathrm{~T} \%$ & Sc & $\mathrm{Cr}$ & Co & $\mathrm{Zn}$ & As & $\mathrm{Rb}$ & $\mathrm{Sb}$ & Cs & $\mathrm{Ba}$ & $\mathrm{La}$ & $\mathrm{Ce}$ & $\mathrm{Nd}$ & $\mathrm{Sm}$ & $\mathrm{Eu}$ & $\mathrm{Tb}$ & $\mathrm{Yb}$ & Lu & $\mathrm{Hf}$ & Ta \\
\hline QR-2 & $51 \mathrm{c}$ & .35 & .06 & 62 & 12.7 & 61.3 & 6.66 & 69.5 & 9.12 & 187 & 0.95 & 13.4 & 436 & 33.5 & & & & 0.97 & 0.82 & 2.70 & & 6.70 & 1.80 \\
\hline QR-3 & $51 c$ & 0.97 & 37 & 5.58 & 13.7 & 2.5 & 8.28 & 77.4 & 2.32 & 188 & 0.72 & 8.50 & 151 & 40.8 & 83.8 & 39.2 & 7.34 & 1.34 & 1.03 & 3.28 & 0.48 & 6.87 & 1.85 \\
\hline QR-4 & $51 c$ & 0.46 & .18 & 6.58 & 13.4 & 65.5 & 6.08 & 67.6 & 5.44 & 203 & 0.73 & 15.4 & 468 & 34.9 & 67.0 & 32.4 & 5.94 & 1.07 & 0.82 & 2.61 & 0.40 & 5.32 & 1.75 \\
\hline QR-5 & A51c & 0.52 & 69 & 6.39 & 14.2 & 74.8 & 9.08 & 85.2 & 3.27 & 222 & 0.90 & 15.9 & 453 & 44.5 & 89.2 & 42.7 & 8.19 & 1.39 & 0.99 & 3.26 & 0.50 & 7.77 & 1.95 \\
\hline QR-6 & $51 \mathrm{c}$ & 0.63 & .05 & 6.67 & 14.5 & 72.7 & 8.54 & 94.7 & 7.56 & 234 & 0.96 & 15.1 & 549 & 40.5 & 79.1 & 38.7 & 7.51 & 1.38 & 0.93 & 3.24 & 0.47 & 5.27 & 1.91 \\
\hline QR-7 & A51c & 0.37 & 3.38 & 6.64 & 15.2 & 70.5 & 7.97 & 82.5 & 7.61 & 227 & 0.95 & 16.3 & 444 & 45.5 & 87.3 & 46.3 & 8.96 & 1.71 & 1.25 & 3.92 & 0.55 & 5.84 & 1.89 \\
\hline QR-8 & A51c & 0.43 & 32 & 5.17 & 12.9 & 61.4 & 7.78 & 75.2 & 11.9 & 192 & 0.83 & 12.4 & 423 & 37.8 & 77.4 & 37.1 & 7.82 & 1.38 & 1.04 & 2.8 & 0.49 & .12 & 1.62 \\
\hline QR-9 & $51 \mathrm{c}$ & 0.62 & 36 & 6.24 & 13.7 & 66.0 & 10.2 & 88.6 & 9.51 & 197 & 0.82 & 14.2 & 539 & 46.0 & 91.5 & 44.7 & 8.93 & 1.45 & 1.17 & 3.33 & 0.48 & 7.65 & 1.90 \\
\hline QR-10 & A51c & 0.47 & 3.30 & 4.87 & 12.4 & 59.5 & 6.80 & 70.2 & 11.3 & 185 & 0.66 & 12.5 & 380 & 36.9 & 71.9 & 34.9 & 6.85 & 1.12 & 0.88 & 2.73 & 0.41 & 6.23 & 1.69 \\
\hline QR-11 & A51c & 0.61 & .27 & 5.05 & 12.3 & 59.0 & 6.96 & 75.7 & 8.30 & 200 & 0.70 & 12.4 & 414 & 46.0 & 89.9 & 42.7 & 8.51 & 1.35 & 1.08 & 3.14 & 0.5 & 7.52 & 1.92 \\
\hline QR-12 & $51 \mathrm{c}$ & 0.46 & 59 & 5.62 & 14.8 & 67.0 & 9.62 & 96.6 & 4.93 & 216 & 0.86 & 14.7 & 449 & 51.5 & 100 & 51.1 & 10.4 & 1.72 & 1.39 & 4.07 & 0.58 & 3.07 & 2.17 \\
\hline QR-13 & A51c & 0.64 & 4.54 & 6.46 & 15.3 & 68.4 & 8.24 & 83.4 & 15.3 & 213 & 0.95 & 15.8 & 467 & 49.7 & 89.2 & 45.3 & 8.89 & 1.59 & 1.17 & 3.97 & 0.56 & 7.95 & 2.16 \\
\hline QR-14 & A51c & 0.41 & 3.60 & 5.99 & 14.4 & 62.7 & 7.66 & 88.5 & 6.08 & 222 & 0.91 & 15.7 & 431 & 47.1 & 91.1 & 45.7 & 9.14 & 1.54 & 1.18 & 3.99 & 0.61 & 8.35 & 2.03 \\
\hline QR-15 & $51 \mathrm{c}$ & 0.47 & 85 & 5.92 & 13.5 & 60.9 & 8.04 & 91.4 & 4.77 & 226 & 0.93 & 14.5 & 441 & 48.0 & 5.6 & 45.9 & 16 & 1.46 & 1.13 & 3.58 & 0.57 & .88 & 2.01 \\
\hline QR-16 & A51c & 0.71 & 4.16 & 6.83 & 14.5 & 67.1 & 10.3 & 97.4 & 9.43 & 218 & 0.92 & 14.2 & 527 & 52.0 & 91.5 & 49.7 & 9.50 & 1.71 & 1.26 & 4.06 & 0.61 & 8.40 & 2.14 \\
\hline QR-17 & A51c & 0.80 & 33 & 4.68 & 10.5 & 49.0 & 5.53 & 53.8 & 10.6 & 166 & 0.65 & 10.0 & 605 & 45.3 & & 41.5 & 8.05 & 1.34 & 1.03 & 2.96 & 0.43 & 6.97 & 1.72 \\
\hline QR-18 & $51 c$ & 0.71 & 59 & 4.58 & 12.1 & 59.2 & 7.13 & 78.7 & 5.70 & 188 & 0.70 & 12.9 & 397 & 48.8 & 1.4 & 43.7 & 8.79 & 1.41 & 1.11 & 3.21 & 0.50 & 7.71 & 1.89 \\
\hline QR-19 & A51c & 0.43 & 3.84 & 7.11 & 14.4 & 68.4 & 8.05 & 90.7 & 10.4 & 227 & 0.98 & 15.3 & 397 & 39.5 & 72.4 & 35.9 & 6.45 & 1.11 & 0.89 & 2.95 & 0.43 & 6.49 & 1.90 \\
\hline QR-20 & A51c & 0.50 & 3.53 & 5.15 & 12.4 & 57.7 & 6.66 & 70.1 & 11.5 & 175 & 0.88 & 12.0 & 367 & 43.8 & 78.4 & 38.6 & 7.14 & 1.18 & 0.92 & 2.99 & 0.40 & 6.94 & 1.88 \\
\hline QR-21 & $51 c$ & 0.50 & 99 & 6.41 & 13.3 & 57.0 & 7.93 & 88.8 & 7.65 & 210 & 0.85 & 14.1 & 394 & 45.6 & 6.8 & 37.9 & 93 & 1.33 & 1.00 & 3.19 & 0.48 & 89 & 1.77 \\
\hline QR-22 & A51c & 0.47 & 3.95 & 6.46 & 14.5 & 64.2 & 7.44 & 86.1 & 6.97 & 209 & 1.03 & 14.4 & 425 & 49.2 & 86.2 & 42.8 & 9.09 & 1.54 & 1.20 & 3.93 & 0.57 & 7.27 & 2.01 \\
\hline QR-23 & A51c & 0.55 & 64 & 4.68 & 11.9 & 55.8 & 6.52 & 73.0 & 10.5 & 183 & 0.66 & 12.3 & 345 & 42.3 & 74.2 & 37.4 & 7.68 & 1.36 & 1.05 & 3.06 & 0.47 & 6.32 & 1.68 \\
\hline QR-25 & A51c & 0.78 & 26 & 5.21 & 10.8 & 2.5 & 7.73 & 68.0 & 6.72 & 169 & 0.73 & 10.4 & 346 & 44.9 & 80.1 & 40.2 & 7.95 & 1.39 & 1.00 & 2.97 & 0.45 & 5.67 & 1.63 \\
\hline QR-26 & A51c & 0.73 & .07 & 4.84 & 9.97 & 48.6 & 7.17 & 66.7 & 6.25 & 155 & 0.75 & 9.72 & 319 & 41.4 & 73.7 & 36.7 & 7.43 & 1.31 & 0.93 & 2.82 & 0.44 & 5.07 & 1.49 \\
\hline QR-27 & A51c & 0.47 & 3.70 & 5.69 & 13.1 & 59.7 & 9.08 & 89.0 & 8.31 & 208 & 0.78 & 13.5 & 389 & 43.3 & 80.0 & 40.3 & 8.26 & 1.45 & 1.10 & 3.51 & 0.49 & 6.58 & 1.79 \\
\hline QR-28 & 151c & 0.60 & 81 & 5.92 & 4.1 & 60.3 & 10.1 & 98.0 & 4.80 & 219 & 0.84 & 14.3 & 394 & 60.3 & 108 & 58.0 & 11.8 & 2.02 & 1.52 & 4.51 & 0.65 & .18 & 1.98 \\
\hline QR-29 & A51c & 0.49 & 61 & 4.09 & 11.5 & 54.1 & 7.05 & 76.0 & 4.99 & 174 & 0.59 & 11.6 & 336 & 41.3 & 74.4 & 37.2 & 7.61 & 1.14 & 0.93 & 3.01 & 0.47 & 39 & 1.59 \\
\hline QR-30 & A51c & 0.39 & 46 & 4.87 & 11.3 & 54.6 & 9.53 & 88.0 & 7.34 & 186 & 0.60 & 11.5 & 352 & 39.3 & 79.6 & 40.9 & 7.87 & 1.41 & 1.12 & 2.78 & 0.47 & 5.31 & 1.68 \\
\hline QR-31 & A51c & 0.64 & 63 & 5.91 & 12.4 & 55.5 & 14.0 & 95.8 & 4.1 & 225 & 0.78 & 13.2 & 432 & 51.9 & 104 & 57.5 & 11.9 & 2.23 & 1.49 & 3.78 & 0.57 & 5.71 & 1.94 \\
\hline QR-32 & A51c & 0.40 & 59 & 5.91 & 14.4 & 64.5 & 9.45 & 77.9 & 8.38 & 208 & 0.89 & 14.3 & 500 & 41.9 & 80.9 & 38.8 & 7.87 & 1.42 & 1.04 & 3.45 & 0.55 & .14 & 2.03 \\
\hline QR-33 & A51c & 0.44 & 3.30 & 5.39 & 12.2 & 57.6 & 5.81 & 68.4 & 11.3 & 176 & 0.70 & 11.3 & 568 & 37.8 & 68.3 & 34.7 & 6.86 & 1.21 & 0.91 & 2.87 & 0.44 & 5.89 & 1.66 \\
\hline QR-34 & A51c & 0.63 & 64 & 5.65 & 12.2 & 58.5 & 9.82 & 80.5 & & 184 & 0.82 & 11.4 & 415 & 46.5 & & 44.5 & 77 & 1.53 & 1.16 & 3.58 & 0.5 & 5.42 & 1.80 \\
\hline QR-35 & A51c & 0.31 & 74 & 4.29 & 11.8 & 3.1 & 5.36 & 56.4 & 08 & 166 & 0.85 & 12.3 & 352 & 45.9 & 2.4 & 38.8 & 7.21 & 1.20 & 0.94 & 3.02 & 0.41 & .06 & 2.02 \\
\hline QR-36 & A51c & 0.44 & 3.59 & 6.08 & 13.5 & 60.9 & 8.23 & 84.1 & 7.30 & 221 & 0.95 & 14.8 & 400 & 43.4 & 84.3 & 41.0 & 7.82 & 1.33 & 1.08 & 3.14 & 0.51 & 7.68 & 1.91 \\
\hline QR-37 & A51c & 0.49 & 50 & 6.40 & 13.4 & 62.1 & 9.16 & 93.1 & 6.7 & 223 & 0.83 & 13.7 & 392 & 43.0 & & 42.2 & 8. & 1.41 & 1.11 & 3.25 & 0.50 & .87 & 1.85 \\
\hline QR-38 & A51c & 0.58 & 17 & 4.87 & 1.0 & 2.0 & 6.85 & 71.2 & 1 & 191 & 0.65 & 11.3 & 347 & 38.9 & .9 & 39.3 & 31 & 1.22 & 0.97 & 2.72 & 0.40 & .09 & 1.69 \\
\hline QR-39 & A51c & 0.47 & 50 & 5.97 & 13.4 & 60.3 & 8.07 & 90.0 & 9.6 & 214 & 0.89 & 13.7 & 421 & 41.8 & .3 & 40.2 & 7.83 & 1.37 & 1.00 & 3.21 & 0.48 & 5.64 & 1.72 \\
\hline QR-40 & A51c & 0.45 & 34 & 5.59 & 12.9 & 57.4 & 8.30 & 79.6 & 7.8 & 205 & 0.84 & 13.4 & 598 & 41.7 & .7 & 43.3 & 8.76 & 1.50 & 1.16 & 3.44 & 0.5 & .95 & 1.79 \\
\hline QR-169 & A51c & 0.44 & 10 & 5.37 & 2.4 & 4.4 & 8.22 & 85.4 & 33 & 197 & 0.75 & 12.3 & 362 & 51.8 & 3.9 & 58.4 & 11.4 & 1.91 & 1.36 & 3.83 & 0.59 & .17 & 1.81 \\
\hline QR-170 & $51 \mathrm{c}$ & 0.36 & 64 & 7.59 & 14.9 & 68.6 & 10.0 & 97.8 & 9.76 & 230 & 0.88 & 14.8 & 433 & 38.4 & .5 & 37.6 & 6.64 & 1.39 & 1.03 & 3.26 & 0.5 & 69 & 1.77 \\
\hline QR-171 & $51 c$ & 0.40 & 67 & 6.83 & 13.6 & 62.8 & 7.51 & 84.4 & 11.8 & 225 & 1.00 & 14.9 & 375 & 38.0 & 68.6 & 33.1 & 6.71 & 1.04 & 0.83 & 2.82 & 0.45 & 5.90 & 2.66 \\
\hline QR-172 & $51 \mathrm{c}$ & 0.41 & 47 & 6.37 & 12.6 & 3.8 & 7.44 & 77.9 & 9.24 & 207 & 0.89 & 13.4 & 377 & 36.5 & 72.6 & 35.6 & 7.23 & 1.14 & 0.97 & 3.07 & 0.44 & 19 & 1.75 \\
\hline QR-173 & $51 \mathrm{c}$ & 0.47 & .27 & 5.37 & 11.9 & 52.0 & 7.04 & 68.5 & 5.99 & 194 & 0.70 & 13.6 & 362 & 44.1 & .7 & 39.4 & 7.82 & 1.14 & 0.97 & 3.19 & 0.48 & 63 & 1.86 \\
\hline QR-174 & 51c & 0.51 & 44 & 5.54 & 12.0 & & 8.53 & 70.1 & 7.43 & 203 & 0.68 & 14.0 & 35 & 45.5 & & 41.1 & 16 & 1.16 & 1.01 & 3.16 & 0.5 & .96 & 2.04 \\
\hline QR-175 & $51 c$ & 0.37 & 44 & 6.95 & 3.8 & .4 & 6.78 & 78.3 & 10.8 & 217 & 0.81 & 13.7 & 564 & 33.9 & 5.0 & 34.9 & 5.84 & 1.13 & 0.88 & 2.97 & 0.4 & .82 & 1.76 \\
\hline QR-176 & $51 \mathrm{c}$ & 0.51 & .49 & 5.00 & 11.1 & 1.1 & 7.29 & 71.3 & 5.97 & 183 & 0.63 & 11.6 & 357 & 42.8 & 82.9 & 45.8 & 9.01 & 1.29 & 0.99 & 3.08 & 0.45 & .98 & 1.68 \\
\hline QR-177 & $51 c$ & 0.67 & 37 & 5.38 & 12.1 & 56.3 & 7.13 & 73.6 & 7.3 & 189 & 0.76 & 11.6 & 435 & 43.7 & 82.4 & 42.4 & 3.29 & 1.35 & 1.09 & 3.28 & 0.4 & .75 & 1.77 \\
\hline QR-178 & 51c & & & 5.26 & & & 5.23 & 56.7 & & 182 & 0.77 & 12.6 & 526 & & 79.2 & 38.2 & & 1.09 & 0.99 & 3.27 & & .58 & 2.00 \\
\hline QR-179 & A51c & 0.42 & 3.47 & 5.63 & 12.9 & 60.9 & 7.75 & 85.8 & 9.04 & 206 & 0.72 & 13.4 & 345 & 41.6 & 81.0 & 44.8 & 8.11 & 1.43 & 1.04 & 3.28 & 0.46 & 6.12 & 1.76 \\
\hline & & & & & & & & & & 243 & 96 & & & & & & & 1.28 & 1.01 & 3.05 & & & \\
\hline QR-42 & A50 & 0.62 & 64 & 6.19 & 4.5 & & 8.44 & 87.8 & 8.28 & 242 & 0.93 & 15.5 & 457 & 43.6 & .7 & 39.6 & 7.8 & 1.30 & 1.02 & 3.14 & 0.5 & 7.35 & 1.87 \\
\hline QR-43 & A50 & 0.64 & 57 & 6.86 & 15.2 & 69.4 & 8.20 & 84.7 & 6.26 & 233 & 1.08 & 16.1 & 439 & 47.0 & 94.0 & 48.3 & 9.21 & 1.58 & 1.27 & 3.61 & 0.57 & 7.15 & 1.87 \\
\hline QR-44 & 450 & 0.36 & 33 & 4.67 & 11.5 & & 6.24 & 62.9 & 7.89 & 180 & 0.68 & 12.2 & 389 & 35.4 & 72.3 & 35.9 & 6.94 & 1.25 & 0.86 & 2.87 & 0.3 & 5.27 & 1.48 \\
\hline QR-45 & A50 & 0.39 & & 6.02 & 13.5 & & 7.27 & 78.1 & 5.72 & 186 & 0.77 & 12.7 & 475 & 39.9 & 80.3 & 39.8 & 7.57 & 1.33 & 1.01 & 3.31 & 0.4 & 5.83 & 1.92 \\
\hline QR-46 & A50 & 0.41 & 39 & 6.86 & 14.5 & 64.6 & 9.81 & 59.1 & 17.5 & 187 & 1.10 & 13.6 & 554 & 38.4 & 77.9 & 38.3 & 8.00 & 1.30 & 1.07 & 3.32 & 0.49 & .21 & 1.98 \\
\hline QR-47 & A50 & 0.60 & .49 & 5.87 & 13.7 & 62.4 & 10.1 & 95.1 & 6.61 & 221 & 0.81 & 14.0 & 414 & 50.5 & 103 & 53.5 & 10.6 & 1.84 & 1.35 & 3.89 & 0.56 & 7.22 & 1.96 \\
\hline QR-48 & A50 & 0.67 & .26 & 4.26 & 11.2 & & & 79.7 & & 181 & 0.77 & 10.6 & 410 & 46.2 & & 44.8 & 9.11 & 1.56 & 1.19 & 3.37 & 0.4 & 7.51 & 1.93 \\
\hline QR-49 & A50 & 0.69 & 02 & 4.59 & 1.5 & 63.3 & 6.81 & 67.3 & 6.94 & 179 & 0.85 & 11.8 & 417 & 41.5 & 3.3 & 39.7 & 7.42 & 1.15 & 0.96 & 2.85 & 0.45 & .26 & 1.81 \\
\hline QR-50 & A50 & 0.41 & .54 & 7.60 & 16.8 & 76.5 & 9.62 & 99.1 & 6.95 & 218 & 0.96 & 15.2 & 450 & 48.8 & 5.5 & 47.3 & 9.60 & 1.76 & 1.29 & 4.06 & 0.58 & .93 & 2.47 \\
\hline QR-51 & 450 & 0.59 & & 7.02 & & & 11.5 & 94.6 & 8.07 & 253 & 1.01 & 16.3 & 492 & 47.1 & & 47.0 & & 1.70 & 1.27 & 4.07 & 0.5 & .13 & 2.12 \\
\hline QR-53 & A50 & 0.27 & 39 & 6.01 & 13.0 & 63.7 & 7.33 & 84.3 & 11.6 & 182 & 0.87 & 13.1 & 322 & 33.7 & 59.1 & 28.5 & 5.27 & 1.01 & 0.76 & 2.66 & 0.40 & .45 & 1.55 \\
\hline QR-54 & A50 & 0.49 & .17 & 6.78 & 14.4 & 66.5 & 9.08 & 103 & 11.2 & 221 & 1.05 & 15.3 & 391 & 44.0 & 77.9 & 39.4 & 7.28 & 1.24 & 0.99 & 3.05 & 0.48 & 5.04 & 1.84 \\
\hline QR-55 & A50 & 0.51 & .81 & 6.38 & 13.0 & 59.7 & 7.65 & 92.3 & 8.83 & 207 & 0.93 & 13.8 & 397 & 38.5 & 69.1 & 29.0 & 6.79 & 1.16 & 0.89 & 2.90 & 0.4 & 5.32 & 1.67 \\
\hline QR-56 & A50 & 0.57 & 12 & 6.11 & 14.4 & & 8.22 & 93.5 & 8.09 & 229 & 0.94 & 15.2 & 430 & 47.4 & .1 & 39.9 & 7.54 & 1.22 & 1.03 & 3.31 & 0.5 & 7.29 & 1.97 \\
\hline QR-57 & A50 & 0.49 & 13 & 4.61 & 14.5 & 65.0 & 4.07 & 65.3 & 10.3 & 203 & 0.65 & 15.2 & 514 & 45.3 & 58.4 & 32.1 & 8.18 & 1.85 & 1.17 & 3.52 & 0.52 & 5.06 & 1.65 \\
\hline QR-58 & A50 & 0.51 & .76 & 6.17 & 13.1 & 58.8 & 7.74 & 84.0 & & 218 & 0.84 & 13.5 & 414 & 40.4 & 78.6 & 39.0 & 8.03 & 1.37 & 1.15 & 3.57 & 0.5 & 5.65 & 1.81 \\
\hline QR-59 & A50 & 0.49 & 1.02 & 7.52 & 15.3 & 72.9 & 8.74 & 98.0 & 9.65 & 244 & 0.93 & 16.0 & 433 & 43.1 & 77.5 & 36.9 & 7.65 & 1.35 & 1.05 & 3.46 & 0.50 & 5.32 & 1.99 \\
\hline QR-60 & A50 & 0.50 & 63 & 5.37 & 13.3 & 61.7 & 7.76 & 91.0 & 7.31 & 193 & 0.66 & 12.6 & 419 & 44.2 & 78.8 & 40.0 & 8.05 & 1.37 & 0.98 & 3.28 & 0.50 & 5.27 & 1.83 \\
\hline QR-61 & A50 & 0.54 & 3.82 & 6.05 & 14.2 & 61.0 & 9.53 & 94.5 & 7.07 & 214 & 0.79 & 14.0 & 422 & 62.1 & 110 & 61.8 & 12.7 & 2.22 & 1.58 & 4.58 & 0.63 & 8.12 & 2.02 \\
\hline QR-62 & A50 & 0.55 & 1.00 & 6.12 & 14.0 & 60.9 & 9.46 & 92.5 & 5.95 & 220 & 0.93 & 14.9 & 386 & 62.9 & 112 & 60.9 & 12.0 & 2.08 & 1.56 & 4.73 & 0.67 & 3.90 & 2.14 \\
\hline QR-63 & A50 & 0.61 & 3.53 & 5.17 & 11.7 & 54.4 & 7.64 & 74.0 & 3.76 & 202 & 0.73 & 12.4 & 385 & 38.8 & 79.8 & 40.1 & 7.58 & 1.39 & 0.99 & 3.01 & 0.51 & 6.33 & 1.72 \\
\hline QR-64 & A50 & 0.42 & 3.28 & 6.98 & 14.7 & 66.9 & 11.3 & 107 & 8.70 & 198 & 0.84 & 12.9 & 405 & 51.5 & 103 & 55.8 & 10.8 & 1.94 & 1.41 & 4.04 & 0.66 & 8.15 & 2.19 \\
\hline QR-65 & A50 & 0.33 & 3.26 & 6.23 & 13.1 & 63.7 & 7.80 & 85.8 & 9.63 & 194 & 0.87 & 13.1 & 383 & 37.1 & 70.6 & 34.0 & 6.59 & 1.19 & 0.93 & 3.03 & 0.46 & 5.79 & 1.76 \\
\hline QR-66 & A50 & 0.37 & 3.75 & 7.60 & 15.9 & 79.3 & 8.40 & 99.4 & 13.1 & 215 & 1.11 & 15.1 & 472 & 42.3 & 78.7 & 38.6 & 7.28 & 1.34 & 1.06 & 3.44 & 0.53 & 7.13 & 2.05 \\
\hline
\end{tabular}


Table 3 (continued)

\begin{tabular}{|c|c|c|c|c|c|c|c|c|c|c|c|c|c|c|c|c|c|c|c|c|c|c|c|c|c|}
\hline Sample & Type & $\mathrm{Na}_{2} \mathrm{O} \%$ & $\mathrm{~K}_{2} \mathrm{O} \%$ & $\mathrm{Fe}_{2} \mathrm{O}_{3} \mathrm{~T} \%$ & Sc & $\mathrm{Cr}$ & Co & Zn & As & $\mathrm{Rb}$ & $\mathrm{Sb}$ & Cs & $\mathrm{Ba}$ & La & $\mathrm{Ce}$ & $\mathrm{Nd}$ & $\mathrm{Sm}$ & $\mathrm{Eu}$ & $\mathrm{Tb}$ & $\mathrm{Yb}$ & Lu & $\mathrm{Hf}$ & Та & Th & U \\
\hline QR-67 & A50 & 0.48 & 4.16 & 6.31 & 13.4 & 58.5 & 8.14 & 87.5 & 8.57 & 222 & 0.97 & 14.1 & 444 & 43.9 & 82.6 & 41.8 & 8.30 & 1.51 & 1.21 & 3.60 & 0.56 & 6.16 & 1.76 & 15.7 & 3.90 \\
\hline QR-68 & A50 & 55 & 3.73 & 6.70 & 14.4 & 5.7 & 9.21 & 95.9 & 10.8 & 219 & 0.92 & 14.5 & 447 & 42.6 & 79.1 & 39.7 & 7.79 & 1.39 & 1.10 & 3.33 & 0.53 & 69 & 1.79 & 5.5 & 3.70 \\
\hline QR-69 & A50 & 0.50 & 3.56 & 7.07 & 14.5 & 64.9 & 8.27 & 92.8 & 8.63 & 242 & 0.93 & 15.1 & 391 & 40.4 & 78.4 & 38.8 & 7.64 & 1.34 & 1.06 & 3.27 & 0.48 & 6.19 & 1.97 & 16.2 & 3.58 \\
\hline QR-70 & A50 & 0.66 & 3.14 & 5.02 & 11.6 & 52.9 & 7.32 & 71.4 & 7.80 & 202 & 0.82 & 12.6 & 375 & 39.8 & 79.4 & 40.1 & 7.73 & 1.36 & 0.99 & 3.13 & 0.46 & .58 & 1.72 & 16.2 & 2.98 \\
\hline QR-71 & A50 & 0.51 & 3.83 & 7.20 & 14.8 & 67.2 & 8.36 & 99.2 & 10.4 & 248 & 0.97 & 15.9 & 432 & 41.9 & 78.9 & 38.1 & 7.81 & 1.39 & 1.10 & 3.29 & 0.47 & .16 & 1.83 & 16.6 & 3.65 \\
\hline QR-72 & A50 & 0.32 & 2.66 & 5.63 & 12.6 & 57.6 & 4.87 & 60.9 & 12.0 & 165 & 0.92 & 12.6 & 421 & 27.2 & 52.3 & 25.9 & 5.19 & 0.93 & 0.72 & 2.48 & 0.38 & 5.68 & 1.65 & 14.5 & 2.96 \\
\hline QR-152 & A50 & 0.31 & 3.48 & 7.05 & 14.9 & 74.7 & 8.47 & 92.4 & 13.6 & 204 & 1.14 & 14.7 & 378 & 36.5 & 68.5 & 31.9 & 6.17 & 1.08 & 0.84 & 2.87 & 0.46 & 5.23 & 1.89 & 16.7 & 2.86 \\
\hline QR-153 & A50 & 0.52 & 3.85 & 6.59 & 14.7 & 67.5 & 9.56 & 103 & 6.74 & 240 & 1.05 & 15.1 & 450 & 41.9 & 84.5 & 44.3 & 8.55 & 1.41 & 1.12 & 3.17 & 0.50 & .39 & 1.88 & 16.5 & 3.35 \\
\hline QR-154 & A50 & 0.35 & 3.71 & 7.07 & 15.7 & 74.2 & 9.35 & 103 & 11.5 & 229 & 1.01 & 16.0 & 449 & 41.0 & 81.8 & 38.8 & 7.39 & 1.42 & 1.09 & 3.44 & 0.54 & .31 & 2.03 & 16.2 & 3.13 \\
\hline QR-155 & A50 & 0.45 & 3.50 & 5.99 & 13.3 & 60.3 & 8.14 & 84.2 & 10.2 & 207 & 0.80 & 13.5 & 422 & 41.2 & 80.9 & 41.0 & 7.70 & 1.41 & 1.06 & 3.31 & 0.48 & .65 & 1.82 & 16.7 & 3.18 \\
\hline QR-156 & A50 & 0.52 & 3.78 & 5.98 & 13.2 & 59.3 & 8.49 & 88.4 & 10.2 & 216 & 0.91 & 14.2 & 403 & 42.5 & 80.7 & 41.5 & 8.03 & 1.32 & 1.05 & 3.28 & 0.50 & 62 & 1.87 & 16.3 & 3.71 \\
\hline QR-157 & A50 & 0.57 & 3.87 & 7.17 & 16.2 & 69.1 & 11.6 & 109 & 7.51 & 239 & 1.04 & 17.2 & 398 & 54.7 & 108 & 56.6 & 11.8 & 2.17 & 1.56 & 4.83 & 0.74 & 7.95 & 2.25 & 19.8 & 3.16 \\
\hline QR-158 & A50 & 0.43 & 3.44 & 5.60 & 14.0 & 60.9 & 7.46 & 77.6 & 10.1 & 196 & 0.79 & 14.3 & 392 & 40.4 & 78.7 & 40.4 & 7.81 & 1.31 & 1.02 & 3.22 & 0.49 & .64 & 1.94 & 16.7 & 3.41 \\
\hline QR-159 & A50 & 0.62 & 3.38 & 5.56 & 13.2 & 59.6 & 8.09 & 83.5 & 7.90 & 200 & 0.83 & 14.0 & 375 & 41.5 & 83.7 & 43.6 & 8.67 & 1.39 & 1.16 & 3.37 & 0.47 & .26 & 1.90 & 15.9 & 3.38 \\
\hline QR-160 & A50 & 0.41 & 3.22 & 6.59 & 14.6 & 2.7 & 8.19 & 77.9 & 8.11 & 196 & 0.97 & 15.0 & 435 & 44.5 & 87.0 & 44.9 & 8.38 & 1.45 & 1.06 & 3.68 & 0.62 & .65 & 1.92 & 18.2 & 3.35 \\
\hline QR-161 & A50 & 0.63 & 3.81 & 6.33 & 14.9 & 65.1 & 8.33 & 94.3 & 6.50 & 223 & 0.79 & 15.1 & 48 & 47.4 & 89.4 & 45.3 & 8.20 & 1.41 & 1.19 & 3.69 & 0.54 & .06 & 2.01 & 18.1 & 3.60 \\
\hline QR-162 & A50 & 0.64 & 3.60 & 6.19 & 13.4 & 59.2 & 11.6 & 87.1 & 9.83 & 216 & 0.90 & 13.8 & & 47.3 & 91.3 & 49.6 & 9.90 & 1.69 & 1.39 & 3.74 & 0.52 & 49 & 1.82 & 16.1 & 4.30 \\
\hline QR- & A50 & 0.44 & 3.61 & 6.16 & 13.6 & 6.8 & 8.56 & 95.7 & 5.86 & 222 & 0.94 & 14.5 & 44 & 47.6 & 92.7 & 48.2 & 9.11 & 1.46 & 1.27 & 4.02 & 0.57 & .82 & 2.27 & 19.1 & 3.91 \\
\hline QR-164 & A50 & 0.43 & 3.30 & 4.78 & 11.9 & 54.1 & 7.51 & 71.2 & 7.45 & 174 & 0.70 & 11.8 & 386 & 35.9 & 75.1 & 36.8 & 5.87 & 1.25 & 0.97 & 2.98 & 0.48 & 5.77 & 1.52 & 13.9 & 2.55 \\
\hline QR-165 & A50 & & 3.75 & 6.69 & & & & 91.1 & 10.9 & 21 & 0.92 & 15.1 & & 39.9 & 6 & 36.0 & 7.07 & 1.22 & 1.02 & 3.04 & 19 & & 1.92 & 16.1 & 3.38 \\
\hline QR-166 & A50 & 55 & 3.74 & 6.96 & 5.0 & .7 & 9.62 & 105 & 13.0 & 225 & 1.00 & 15.2 & 43 & 44.0 & 84.0 & 41.5 & 8.06 & 1.44 & 1.17 & 50 & 47 & 16 & 1.95 & 16.3 & 3.99 \\
\hline QR-167 & A50 & 0.50 & 3.54 & 5.88 & 14.1 & 59.7 & 9.42 & 93.0 & 6.24 & 200 & 0.80 & 13.7 & 421 & 58.4 & 112 & 60.1 & 11.8 & 2.17 & 1.56 & 4.50 & 0.63 & .32 & 1.87 & 19.4 & 3.55 \\
\hline QR-168 & A50 & 0.70 & 3.48 & 4.90 & 12.0 & & 7.35 & 73.5 & 7.50 & 213 & 0.69 & 12.6 & 38 & 44.8 & 84.6 & 42.0 & 8.67 & 1.46 & 1.09 & 3.24 & 0.50 & 57 & 1.65 & 16.4 & 2.98 \\
\hline QR-180 & A50 & 0.44 & 3.02 & 5.43 & 12.6 & 5.2 & 8.65 & 184 & 5.46 & 175 & 0.84 & 12.4 & 382 & 52.2 & 103 & 58.0 & 11.4 & 2.00 & 1.35 & 3.93 & 0.58 & 7.37 & 1.88 & 17.6 & 3.19 \\
\hline & L9 & & & & & & & & 8.67 & 208 & & & & 50.7 & & & & & 1.18 & & & & & 17.8 & \\
\hline QR- & L9 & & 41 & 7.78 & & & 9.76 & 105 & 13.1 & 244 & 1.00 & 6.10 & & 44.2 & & 37.5 & & 1.26 & 0.95 & & & & 39 & 15.7 & 3.45 \\
\hline & L9 & & & & & & & & & & & 12.9 & & & & & & & 1.08 & & & & & 15.1 & \\
\hline QR-75 & L9 & 0.46 & 4.28 & 7.83 & 14.6 & 7.1 & 9.28 & 104 & 14.7 & 225 & 0.99 & 14.7 & & 42.8 & 3 & 39.3 & 7.67 & 1.40 & 1.13 & 50 & & 34 & 1.90 & 16.5 & 3.56 \\
\hline QR-76 & L9 & & 4.28 & 5.81 & & & & 92.7 & 5.86 & 219 & 0.91 & 13.7 & & 45.8 & & & & 1.42 & 1.08 & & & 77 & 1.71 & 15.9 & \\
\hline QR-77 & L9 & & 4.59 & & & & & 104 & & 24 & 1.08 & & & & & & & & 1.33 & & & & 1 & 17.3 & \\
\hline QR-78 & L9 & 0.67 & 3.49 & 4.93 & 1.2 & 1.0 & 9.87 & 82.8 & 5.55 & 181 & 0.72 & 11.3 & 32 & 41.3 & 79.6 & 42.8 & 8.24 & 1.47 & 1.06 & 3.09 & 0.50 & 78 & 1.55 & 14.2 & 2.72 \\
\hline QR-79 & L9 & & 4.22 & 6.58 & & & & 93.0 & 9.11 & 213 & 0.99 & 13.4 & & 46.9 & & 40.3 & & 1.44 & 1.14 & & & 27 & 1.84 & 15.2 & 3.81 \\
\hline QR-80 & L9 & & 3.72 & & & & & 77. & 7.86 & 191 & 0.79 & & & & & & & & 1.10 & & & & 1.64 & 14.5 & \\
\hline QR-81 & L9 & 45 & 3.71 & 6.79 & 4.0 & .9 & 7.38 & 79.1 & 9.38 & 207 & 0.96 & 15.0 & 38 & 41.5 & 1 & 16.8 & 6.87 & 1.18 & 0.80 & 2.89 & 0.45 & 0 & 1.81 & 15.9 & 3.09 \\
\hline QR-82 & L9 & & 4.32 & 6.77 & & & 9.42 & 97.7 & 8.83 & 230 & 0.46 & 14.1 & & 48.3 & & 42.0 & & 1.50 & 1.21 & & & .87 & 1.88 & 16.5 & 4.23 \\
\hline QR-83 & L9 & & 3.97 & & & & & 86.5 & 14.2 & 193 & 0.18 & & & 40.3 & & & & 1.27 & 0.95 & & & & 1.68 & 14.5 & \\
\hline QR-84 & L9 & 30 & 3.30 & 7.09 & 14.5 & 66.8 & 8.39 & 89.7 & 7.97 & 229 & 0.96 & 15.6 & 43 & 37.3 & 75.9 & 35.4 & 6.59 & 1.13 & 0.87 & 2.97 & 0.52 & 81 & 2.08 & 17.4 & 3.15 \\
\hline QR-85 & L9 & & 3.76 & 7.72 & & 68.8 & & 101 & 10.6 & 247 & 0.92 & 15.5 & & 39.1 & 77.8 & 38.9 & & 1.38 & 1.03 & 3.1 & 0. & 58 & 1.88 & 15.4 & 3.43 \\
\hline QR-86 & L9 & & 3.22 & 5.52 & & 54.8 & & 74.5 & 8.04 & 183 & 0.80 & 12.0 & & 36.2 & & 35.2 & & 1.14 & 0.95 & & & 33 & 1.71 & 15.4 & 3.29 \\
\hline QR-87 & L9 & 0.41 & 3.68 & 8.02 & 15.6 & 72.2 & 10.5 & 108 & 10.9 & 255 & 0.97 & 16.0 & 44 & 37.4 & 78.6 & 40.5 & 7.67 & 1.47 & 1.04 & 3.38 & 0.55 & .97 & 1.96 & 15.5 & 3.57 \\
\hline QR-88 & L9 & & 2.83 & 6.15 & & & & 65.7 & 11.4 & 164 & 0.80 & 11.3 & & 35.0 & 69.5 & 33.9 & 6.34 & 0.97 & 0.78 & 2.6 & 0. & 7.44 & 1.66 & 17.2 & 3.32 \\
\hline QR-89 & L9 & & 3.41 & & & & & 92.4 & 10.0 & 227 & 0.91 & & & 36.2 & & & & 1.33 & 0.99 & & & & 1.80 & 15.9 & 2.82 \\
\hline QR-90 & L9 & 0.31 & 3.31 & 7.52 & 15.2 & 75.0 & 9.52 & 87.6 & 16.2 & 214 & 1.17 & 15.1 & 83 & 35.6 & 70.5 & 33.5 & 6.69 & 1.20 & 0.93 & 3.19 & 0.51 & 6.48 & 1.98 & 17.3 & 3.96 \\
\hline QR-91 & L9 & & 2.97 & 7.00 & & & 6.33 & 80.7 & 11.5 & 201 & 1.00 & 13.1 & & 38.1 & 69.4 & 35.6 & 7.34 & 1.26 & 1.02 & 3.3 & 0. & .21 & 1.87 & 16.9 & 3.60 \\
\hline QR-92 & L9 & & 3.85 & 6.54 & & 60. & 9.22 & 94.6 & 7.68 & 229 & 1.02 & 13.9 & & 42.6 & & 41.0 & 3 & 1.50 & 1.20 & 3.44 & 0. & 75 & 1.81 & 16.2 & 3.45 \\
\hline QR-141 & L9 & 0.33 & 2.76 & 4.64 & 10.8 & 49.6 & 5.36 & 59.9 & 8.84 & 156 & 0.74 & 9.53 & 444 & 27.8 & 53.7 & 26.5 & 5.50 & 0.96 & 0.79 & 2.57 & 0.40 & 5.53 & 1.43 & 13.5 & 2.36 \\
\hline QR-142 & L9 & 0.45 & 3.43 & 7.45 & 14.4 & 68.9 & 10.0 & 101 & 9.27 & 228 & 0.91 & 13.8 & 422 & 39.9 & 76.7 & 39.2 & 7.66 & 1.36 & 1.01 & 3.22 & 0.49 & 5.98 & 1.79 & 15.8 & 2.95 \\
\hline & L9 & & 3.31 & & & & & & 9.86 & 202 & 0.83 & 13.0 & & 36.1 & 71.2 & 34.5 & 7.00 & 1.24 & 0.95 & 3.14 & & 6.46 & 1.79 & 15.9 & 3.65 \\
\hline QR-144 & L9 & 0.47 & 3.14 & 5.84 & 11.7 & 53.3 & 7.85 & 83.7 & 6.96 & 200 & 0.87 & 12.4 & 360 & 37.6 & 73.5 & 37.4 & 7.21 & 1.28 & 1.07 & 3.12 & 0.50 & 5.98 & 1.62 & 14.8 & 3.18 \\
\hline QR-145 & L9 & 0.41 & 3.27 & 7.33 & 13.9 & 65.6 & 9.81 & 94.8 & 9.56 & 216 & 0.91 & 13.3 & 369 & 41.2 & 101 & 45.7 & 7.74 & 1.31 & 1.06 & 3.33 & 0.50 & 5.66 & 1.78 & 14.9 & 3.22 \\
\hline QR-146 & L9 & 0.61 & 3.84 & 6.10 & 13.0 & 60.3 & 9.44 & 93.6 & 5.81 & 223 & 0.83 & 13.8 & & 42.6 & 87.5 & 44.8 & 8.24 & 1.49 & 1.12 & 3.35 & 0.5 & 7.08 & 1.88 & 16.5 & 3.72 \\
\hline QR-147 & L9 & 0.40 & 3.65 & 8.24 & 15.3 & 74.1 & 11.0 & 108 & 12.2 & 234 & 1.08 & 14.8 & 486 & 42.6 & 81.3 & 41.6 & 7.76 & 1.45 & 1.12 & 3.33 & 0.50 & 6.09 & 1.84 & 16.4 & 3.20 \\
\hline QR-148 & L9 & 0.45 & 3.53 & 6.29 & 12.8 & 56.4 & 8.63 & 87.8 & 8.04 & 214 & 1.01 & 13.4 & 395 & 39.1 & 77.7 & 41.1 & 8.03 & 1.41 & 1.06 & 3.25 & 0.48 & 5.98 & 1.71 & 14.9 & 3.80 \\
\hline QR-149 & L9 & & 3.35 & 7.24 & 13.5 & 57.5 & 7.87 & 94.8 & 13.8 & 220 & 1.01 & 15.1 & 59 & 41.1 & 76.5 & 39.0 & 7.97 & 1.30 & 1.02 & 3.20 & 0.49 & 5.85 & 1.80 & 16.5 & 3.60 \\
\hline QR-150 & L9 & 0.28 & 2.33 & 3.53 & 8.24 & 39.3 & 5.03 & 48.9 & 4.90 & 126 & 0.47 & 8.25 & 257 & 26.1 & 52.9 & 26.3 & 4.90 & 0.95 & 0.68 & 2.01 & 0.31 & 4.29 & 1.23 & 10.4 & 2.22 \\
\hline QR-151 & L9 & 0.55 & 2.80 & 4.65 & 10.8 & 50.3 & 7.01 & 80.2 & 5.33 & 167 & 0.76 & 10.3 & 320 & 36.9 & 73.5 & 36.5 & 6.62 & 1.19 & 0.88 & 2.92 & 0.40 & 6.52 & 1.60 & 15.0 & 2.38 \\
\hline
\end{tabular}

(PCA) (non-supervised) and discriminate analysis (supervised) transformation provided a new basis for viewing the entire data distribution, allowing a better grasp of its structure. Bivariate and trivariate scatter plots were also used for a better scanning of the correlations between variables, also contributing to the detection of obvious groups and of outliers. For this statistical approach, the absolute concentrations of elements, as well as the normalized contents relative to the Sc content ([X]sample/[Sc]sample), were used as variables. This normalization procedure was done prior to any further statistical approach. Comparative studies of ceramics produced with similar raw materials, as are the ones from the Tagus estuary, must pass through a first step of normalization, especially because the temper is mainly composed of quartz, diluting chemical differences; normalization being a procedure allowing to compensate for the influence of natural processes on the measured variability of the concentrations of elements (Dias and Prudêncio, 2008). As scandium is a conservative element, it is most resistant and tends to survive in superficial environments during weathering; as such, it will not be leached and carried away easily during ancient ceramic burial. Another important argument for the choice of this element is that its concentration is obtained with good precision and accuracy by INAA.

\section{Results and discussion}

A description of the geochemical patterns of each production centre, taken separately, will be presented first, followed by a discussion comparing the two centres, belonging to the same 
Table 4

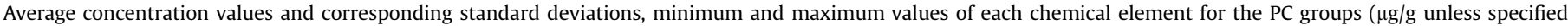
otherwise).

\begin{tabular}{|c|c|c|c|c|c|c|c|c|c|c|c|c|c|c|c|}
\hline & \multicolumn{5}{|c|}{ GROUP 1} & \multicolumn{5}{|c|}{ GROUP 2} & \multicolumn{5}{|c|}{ GROUP 3} \\
\hline & $n$ & $\bar{x}$ & Min. & Max. & $\sigma$ & $n$ & $\bar{x}$ & Min. & Max. & $\sigma$ & $n$ & $\bar{x}$ & Min. & Max. & $\sigma$ \\
\hline $\mathrm{Na}_{2} \mathrm{O}, \%$ & 38 & 0.434 & 0.250 & 0.670 & 0.0785 & 24 & 0.686 & 0.510 & 0.820 & 0.0887 & 61 & 0.596 & 0.370 & 0.790 & 0.0979 \\
\hline $\mathrm{K}_{2} \mathrm{O}, \%$ & 37 & 3.29 & 3.03 & 3.36 & 0.1617 & 24 & 3.25 & 2.81 & 3.520 & 0.1741 & 60 & 3.27 & 2.78 & 3.84 & 0.2279 \\
\hline $\mathrm{Fe}_{2} \mathrm{O}_{3} \mathrm{~T}, \%$ & 39 & 4.71 & 4.27 & 4.91 & 0.2665 & 24 & 4.76 & 3.92 & 5.86 & 0.5120 & 56 & 4.65 & 3.96 & 5.37 & 0.3018 \\
\hline Sc & 38 & 13.2 & 11.7 & 14.9 & 0.7110 & 24 & 12.3 & 11.0 & 13.8 & 0.6821 & 60 & 13.6 & 11.5 & 15.7 & 0.9596 \\
\hline $\mathrm{Cr}$ & 39 & 64.7 & 54.9 & 75.8 & 4.806 & 24 & 56.7 & 52.1 & 61.6 & 2.8582 & 61 & 65.5 & 52.8 & 78.3 & 5.564 \\
\hline Co & 38 & 15.3 & 10.5 & 20.2 & 2.139 & 21 & 8.71 & 6.97 & 10.7 & 0.9097 & 60 & 9.10 & 6.44 & 13.6 & 1.832 \\
\hline $\mathrm{Zn}$ & 39 & 78.5 & 67.0 & 88.2 & 5.715 & 24 & 74.0 & 54.1 & 93.4 & 10.18 & 60 & 70.2 & 48.5 & 92.1 & 9.013 \\
\hline As & 39 & 18.8 & 12.7 & 25.1 & 2.785 & 24 & 8.23 & 4.29 & 14.3 & 2.674 & 61 & 16.5 & 4.70 & 28.2 & 5.771 \\
\hline $\mathrm{Rb}$ & 39 & 188 & 168 & 209 & 9.933 & 24 & 180 & 153 & 199 & 10.26 & 60 & 183 & 154 & 208 & 13.10 \\
\hline $\mathrm{Sb}$ & 40 & 0.526 & 0.360 & 0.65 & 0.0638 & 24 & 0.647 & 0.500 & 0.780 & 0.0774 & 61 & 0.565 & 0.390 & 0.780 & 0.0931 \\
\hline Cs & 39 & 13.5 & 11.6 & 15.3 & 0.9134 & 24 & 12.2 & 10.6 & 14.1 & 0.8319 & 60 & 13.6 & 11.3 & 16.4 & 1.179 \\
\hline Ва & 40 & 503 & 392 & 612 & 54.68 & 23 & 459 & 378 & 566 & 48.24 & 59 & 529 & 361 & 700 & 70.71 \\
\hline $\mathrm{La}$ & 40 & 50.5 & 37.7 & 59.5 & 5.425 & 23 & 44.1 & 34.1 & 54.2 & 4.802 & 61 & 42.1 & 26.6 & 58.7 & 6.610 \\
\hline $\mathrm{Ce}$ & 39 & 83.1 & 63.3 & 99.0 & 8.621 & 24 & 83.3 & 67.6 & 101 & 7.993 & 61 & 79.6 & 53.5 & 112 & 12.62 \\
\hline $\mathrm{Nd}$ & 40 & 48.0 & 35.6 & 57.9 & 5.629 & 24 & 39.8 & 31.5 & 47.3 & 3.812 & 59 & 38.0 & $24-0$ & 48.2 & 5.040 \\
\hline $\mathrm{Sm}$ & 40 & 9.97 & 7.22 & 12.9 & 1.385 & 24 & 7.76 & 6.19 & 8.76 & 0.6464 & 58 & 7.74 & 5.50 & 9.58 & 0.8992 \\
\hline $\mathrm{Eu}$ & 40 & 1.83 & 1.22 & 2.31 & 0.2616 & 23 & 1.22 & 1.08 & 1.38 & 0.0768 & 56 & 1.29 & 0.940 & 1.62 & 0.1446 \\
\hline $\mathrm{Tb}$ & 40 & 1.36 & 0.960 & 1.72 & 0.1807 & 24 & 0.988 & 0.810 & 1.19 & 0.0853 & 57 & 0.983 & 0.710 & 1.24 & 0.1286 \\
\hline $\mathrm{Yb}$ & 40 & 3.87 & 3.10 & 4.76 & 0.4131 & 24 & 3.21 & 2.71 & 3.67 & 0.2524 & 58 & 3.15 & 2.64 & 3.66 & 0.2363 \\
\hline $\mathrm{Lu}$ & 40 & 0.590 & 0.450 & 0.700 & 0.0648 & 24 & 0.506 & 0.430 & 0.570 & 0.0336 & 58 & 0.488 & 0.390 & 0.580 & 0.0459 \\
\hline $\mathrm{Hf}$ & 38 & 7.07 & 5.93 & 8.19 & 0.5156 & 24 & 7.59 & 6.42 & 8.82 & 0.6667 & 61 & 6.84 & 5.30 & 8.37 & 0.7840 \\
\hline Тa & 39 & 1.86 & 1.69 & 2.09 & 0.0958 & 22 & 1.78 & 1.69 & 1.92 & 0.0632 & 58 & 1.84 & 1.54 & 2.12 & 0.1257 \\
\hline Th & 40 & 17.3 & 14.7 & 19.7 & 1.111 & 24 & 17.6 & 15.2 & 20.0 & 1.279 & 61 & 16.9 & 13.3 & 19.6 & 1.526 \\
\hline U & 40 & 7.05 & 4.91 & 9.14 & 1.027 & 23 & 4.29 & 3.73 & 4.88 & 0.3032 & 61 & 5.77 & 3.76 & 7.96 & 0.8808 \\
\hline
\end{tabular}

sedimentary basin, in order to establish the chemical tracers for each one and differentiate better between the two.

The chemical data obtained by INAA for the paste composition of amphorae from PC and QR is presented in Tables 2 and 3, respectively.

Samples will be classified into groups of similar elemental composition by exploratory methods (hierarchical cluster analysis, $\mathrm{k}$-means and PCA). The average concentration values and corresponding standard deviations, and the minimum and maximum values of each chemical element for each group are presented in Table 4 for the Porto dos Cacos workshop, in Table 5 for the Quinta do Rouxinol workshop, and in Table 6 for the two workshops analysed comparatively (PC and QR).

The estimated mineralogical composition of selected sherds from the PC and QR workshops is presented in Table 7.

\subsection{Porto dos Cacos workshop}

Cluster analysis is a rapid and efficient technique for evaluating relationships among large numbers of samples between which distance measures have been calculated. The examination of the dendrogram resulting from the cluster analysis of PC amphorae by

Table 5

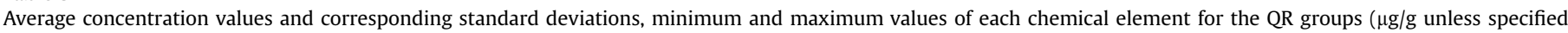
otherwise).

\begin{tabular}{|c|c|c|c|c|c|c|c|c|c|c|}
\hline & \multicolumn{5}{|c|}{ GROUP 1} & \multicolumn{5}{|c|}{ GROUP 2} \\
\hline & $n$ & $\bar{x}$ & Min. & Max. & $\sigma$ & $n$ & $\bar{x}$ & Min. & Max. & $\sigma$ \\
\hline $\mathrm{Na}_{2} \mathrm{O}, \%$ & 27 & 0.461 & 0.306 & 0.661 & 0.0903 & 102 & 0.507 & 0.268 & 0.81 & 0.1183 \\
\hline $\mathrm{K}_{2} \mathrm{O}, \%$ & 27 & 3.67 & 2.76 & 4.59 & 0.5019 & 99 & 3.54 & 2.74 & 4.17 & 0.2958 \\
\hline $\mathrm{Fe}_{2} \mathrm{O}_{3} \mathrm{~T}, \%$ & 27 & 6.82 & 4.64 & 8.24 & 0.8707 & 103 & 5.92 & 3.53 & 7.60 & 0.8855 \\
\hline Sc & 27 & 13.6 & 10.8 & 15.6 & 1.290 & 102 & 13.4 & 9.97 & 16.8 & 1.4372 \\
\hline $\mathrm{Cr}$ & 27 & 63.1 & 49.6 & 75.0 & 6.977 & 101 & 61.3 & 48.6 & 76.5 & 6.319 \\
\hline Co & 27 & 8.74 & 5.36 & 11.1 & 1.553 & 101 & 8.18 & 4.87 & 11.6 & 1.384 \\
\hline $\mathrm{Zn}$ & 27 & 90.7 & 59.9 & 108 & 12.29 & 102 & 83.5 & 48.9 & 109 & 12.60 \\
\hline As & 27 & 10.0 & 5.81 & 16.2 & 2.651 & 101 & 8.08 & 2.32 & 13.6 & 2.260 \\
\hline $\mathrm{Rb}$ & 26 & 217 & 164 & 255 & 20.19 & 102 & 206 & 155 & 222 & 21.62 \\
\hline $\mathrm{Sb}$ & 25 & 0.944 & 0.740 & 1.17 & 0.0988 & 103 & 0.845 & 0.47 & 1.14 & 0.1288 \\
\hline Cs & 26 & 13.9 & 11.3 & 16.1 & 1.213 & 102 & 13.7 & 8.50 & 17.2 & 1.592 \\
\hline $\mathrm{Ba}$ & 25 & 456 & 360 & 624 & 71.05 & 96 & 414 & 319 & 549 & 51.86 \\
\hline La & 26 & 41.2 & 35.0 & 50.7 & 4.330 & 97 & 43.0 & 33.5 & 54.7 & 4.539 \\
\hline $\mathrm{Ce}$ & 24 & 77.7 & 69.1 & 90.6 & 6.186 & 96 & 81.9 & 58.4 & 104 & 9.085 \\
\hline $\mathrm{Nd}$ & 26 & 38.8 & 26.5 & 45.7 & 4.378 & 94 & 40.4 & 26.3 & 55.8 & 5.129 \\
\hline $\mathrm{Sm}$ & 27 & 7.59 & 5.50 & 9.17 & 0.836 & 95 & 7.95 & 5.19 & 10.8 & 1.111 \\
\hline $\mathrm{Eu}$ & 25 & 1.37 & 1.14 & 1.63 & 0.1221 & 93 & 1.35 & 0.930 & 1.84 & 0.1807 \\
\hline $\mathrm{Tb}$ & 27 & 1.04 & 0.780 & 1.33 & 0.1286 & 97 & 1.06 & 0.680 & 1.41 & 0.1470 \\
\hline $\mathrm{Yb}$ & 23 & 3.27 & 2.89 & 3.6 & 0.1556 & 97 & 3.29 & 2.48 & 4.07 & 0.3791 \\
\hline Lu & 25 & 0.513 & 0.450 & 0.580 & 0.0298 & 100 & 0.501 & 0.380 & 0.660 & 0.0602 \\
\hline $\mathrm{Hf}$ & 27 & 6.30 & 5.53 & 7.39 & 0.5171 & 103 & 6.76 & 4.29 & 8.96 & 0.9586 \\
\hline Ta & 26 & 1.81 & 1.62 & 1.98 & 0.0927 & 100 & 1.85 & 1.48 & 2.27 & 0.1690 \\
\hline Th & 27 & 15.9 & 13.5 & 17.8 & 0.9761 & 102 & 16.7 & 13.5 & 20.5 & 1.638 \\
\hline $\mathrm{U}$ & 26 & 3.54 & 2.82 & 4.35 & 0.3786 & 101 & 3.34 & 2.22 & 4.30 & 0.4888 \\
\hline
\end{tabular}


Table 6

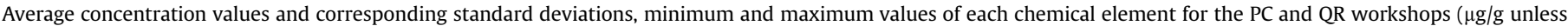
specified otherwise).

\begin{tabular}{|c|c|c|c|c|c|c|c|c|c|c|}
\hline & \multicolumn{5}{|c|}{ Porto dos Cacos } & \multicolumn{5}{|c|}{ Quinta do Rouxinol } \\
\hline & $n$ & $\bar{x}$ & Min. & Max. & $\sigma$ & $n$ & $\bar{x}$ & Min. & Max. & $\sigma$ \\
\hline $\mathrm{Na}_{2} \mathrm{O}, \%$ & 129 & 0.612 & 0.250 & 0.820 & 0.0865 & 129 & 0.497 & 0.268 & 0.810 & 0.1142 \\
\hline $\mathrm{K}_{2} \mathrm{O}, \%$ & 123 & 3.23 & 2.78 & 3.72 & 0.2145 & 127 & 3.56 & 2.66 & 4.46 & 0.3576 \\
\hline $\mathrm{Fe}_{2} \mathrm{O}_{3} \mathrm{~T}, \%$ & 125 & 4.69 & 3.79 & 5.52 & 0.3758 & 130 & 6.11 & 3.53 & 8.24 & 0.9519 \\
\hline $\mathrm{Sc}$ & 129 & 13.2 & 10.5 & 15.7 & 1.110 & 129 & 13.4 & 9.97 & 16.8 & 1.405 \\
\hline $\mathrm{Cr}$ & 128 & 63.4 & 50.0 & 78.3 & 6.553 & 129 & 61.8 & 48.6 & 79.3 & 6.637 \\
\hline Co & 128 & 9.07 & 2.95 & 20.8 & 2.410 & 128 & 8.30 & 4.87 & 11.6 & 1.434 \\
\hline $\mathrm{Zn}$ & 128 & 69.9 & 44.0 & 95.2 & 9.399 & 128 & 85.3 & 53.8 & 109 & 12.47 \\
\hline As & 130 & 15.3 & 4.29 & 34.3 & 5.798 & 128 & 8.49 & 2.32 & 15.3 & 2.453 \\
\hline $\mathrm{Rb}$ & 126 & 183 & 154 & 209 & 12.50 & 129 & 208 & 155 & 255 & 22.16 \\
\hline $\mathrm{Sb}$ & 130 & 0.585 & 0.360 & 0.780 & 0.0960 & 127 & 0.868 & 0.590 & 1.17 & 0.1248 \\
\hline Cs & 128 & 13.3 & 10.1 & 16.4 & 1.272 & 128 & 13.7 & 9.53 & 17.2 & 1.560 \\
\hline $\mathrm{Ba}$ & 126 & 504 & 361 & 676 & 68.44 & 115 & 415 & 319 & 539 & 46.81 \\
\hline $\mathrm{La}$ & 129 & 43.3 & 26.6 & 59.7 & 6.584 & 123 & 42.6 & 33.5 & 54.7 & 4.542 \\
\hline $\mathrm{Ce}$ & 129 & 81.5 & 53.5 & 112 & 10.95 & 119 & 81.2 & 59.1 & 103 & 8.424 \\
\hline Nd & 129 & 39.0 & 24.0 & 57.9 & 5.532 & 117 & 40.1 & 28.5 & 53.5 & 4.491 \\
\hline $\mathrm{Sm}$ & 128 & 7.95 & 4.94 & 12.1 & 1.234 & 117 & 7.83 & 5.50 & 10.4 & 0.8783 \\
\hline $\mathrm{Eu}$ & 130 & 1.29 & 0.460 & 2.31 & 0.2527 & 120 & 1.35 & 0.930 & 1.76 & 0.1797 \\
\hline $\mathrm{Tb}$ & 129 & 1.01 & 0.570 & 1.72 & 0.1522 & 124 & 1.06 & 0.720 & 1.41 & 0.1450 \\
\hline $\mathrm{Yb}$ & 127 & 3.18 & 2.44 & 4.53 & 0.3189 & 124 & 3.28 & 2.48 & 4.07 & 0.3598 \\
\hline Lu & 129 & 0.497 & 0.350 & 0.700 & 0.0549 & 125 & 0.499 & 0.380 & 0.630 & 0.0534 \\
\hline $\mathrm{Hf}$ & 129 & 7.00 & 5.30 & 8.82 & 0.8074 & 129 & 6.64 & 4.29 & 8.90 & 0.8823 \\
\hline Та & 123 & 1.83 & 1.54 & 2.12 & 0.1164 & 127 & 1.84 & 1.48 & 2.27 & 0.1606 \\
\hline Th & 129 & 17.1 & 13.3 & 20.0 & 1.457 & 129 & 16.5 & 13.5 & 20.5 & 1.555 \\
\hline $\mathrm{U}$ & 130 & 5.47 & 3.16 & 9.14 & 1.044 & 128 & 3.37 & 2.22 & 4.35 & 0.4808 \\
\hline
\end{tabular}

Ward's hierarchical clustering method and Euclidean distances, combined with box-and-whisker plot graphs, emphasize the fact that five ceramic samples remain chemically distinct and cannot be attributed to one of the groups. Among these samples, there are two A51c amphorae (PC 17 and PC 19), two A51c/L3? amphorae (PC 2 and PC 40), and one L3 (PC 123) amphora.

These five samples present outliers and extreme values in the chemical distribution, whose range was defined according to the 'classic' box-and-whisker plot. Considering these criteria: (i) PC 17 presents upper extreme values of $\mathrm{Co}$, and upper outlier values of $\mathrm{Zn}$, Hf and Th; (ii) PC 19 presents upper extreme values of K, and upper outlier values of $\mathrm{Na}$, Fe and La; (iii) PC 2 presents upper extreme values of Th; (iv) PC 40 presents upper extreme values of $\mathrm{K}, \mathrm{Cr}, \mathrm{Ce}$, upper outlier values of $\mathrm{Fe}, \mathrm{La}, \mathrm{Nd}, \mathrm{Yb}, \mathrm{Lu}, \mathrm{Hf}$, and lower outlier values of Cs; (v) PC 123 presents upper extreme values of $\mathrm{Zn}$.

Disregarding these five outliers for the further classification and grouping of samples with similar chemical composition, the data analysis points to a certain stratification of the results according to the typology (Figs. 2 and 3). This indicates that, considering the different amphorae shapes, at the PC site most of the L3 amphorae samples are distinct from the others due to their higher contents of REE, especially the heavy ones, $\mathrm{Co}$ and $\mathrm{U}$, and lower values of $\mathrm{Na}$. On the other hand, the majority of the D14 amphorae present higher values of $U$ than the A51c and A51c/L3? amphora types. With regards to the A50 samples, the only conclusion to be drawn is that they do not fit with the L3 amphorae samples, but, due to the reduced number of samples (and also of finds), it is risky to establish a chemical tendency. Nevertheless, these samples are not outliers, and they appear to have been manufactured with the same raw materials used for the remaining PC workshop set.

This tendency of showing only a limited diagnostic chemical compositions for pottery produced at a particular workshop, is a sign of the use of similar raw materials for extended periods of time, and typologically different vessels. Nevertheless, for the PC amphora workshop, three main compositional groups may be defined after applying multivariate statistical tools, one comprising L3 amphorae (Group 1), the other consisting mainly of D14 (together with a few A51c and A51c/L3?) (Group 2), and a third, smaller one (Group 3), including A51c amphorae, together with L3 and A51c/L3? (Fig. 3).

Concerning the doubts about the allocation of the types A51c or L3 (A51c/L3?), we can consider the possibility of the existence of two groups of L3 with distinct composition, which may indicate a variation in the raw materials used over time. Therefore, there is only one group composed exclusively of L3 amphorae (Group 1), and another (Group 3) that includes samples of L3, A51c, and all the A51c/L3? samples whose typology cannot be established with precision (see Fig. 3). Thus, the typological classification of the fragments of amphorae cannot be made according to geochemical criteria, but merely by formal ones. This is because although most of the L3 amphorae have a composition distinct from the other samples, L3 amphorae are also present in another group, comprising the unidentified samples and A51c/L3?, not excluding the possibility of these belonging to the L3 typology. If one considers the analysed samples obtained from the bottoms of amphorae (six L3 and six A51c), and considering the L3 group, one is an outlier, four L3 bottom samples are included in the L3 group (Group 1), and the other in the D14 group (Group 2). Regarding the A51c bottom samples, three are included in Group 2 and the other three in Group 3 (A51c, L3 and A51c/L3?).

In terms of the samples that display potter markings, only one sample (TMM) is included in the D14 group, two samples (AIVNIT (or TINVIA retro); GERMA(ni)) are included in Group 3, and the other samples (RUSTICI; CLARIAMI; GERMAN (retro); CERF (Germanus); and non-identified) are part of Group 1.

This geochemical pattern of amphora pastes reflects, on one hand, the natural inhomogeneity of the raw material, intrinsic to the sedimentary basin, and on the other hand the recourse to diverse clay pits along the sedimentary basin, understandable considering the long period of operation of the workshop.

\subsection{Quinta do Rouxinol workshop}

Cluster analysis was also used in the initial inspection of the source data pertaining to Quinta do Rouxinol amphorae. The resulting dendrogram, using Ward's hierarchical clustering method 
Table 7

Estimated mineralogical composition for selected sherds from the PC and QR workshops (\%).

\begin{tabular}{|c|c|c|c|c|c|c|c|c|c|}
\hline & Type & Sample & Qtz & Phy & Kfs & $\mathrm{Pl}$ & Ant & Hem & Mul \\
\hline & & & 3.34 & 4.48 & 3.25 & 3.20 & 3.52 & 2.69 & 3.39 \\
\hline \multirow[t]{39}{*}{ PORTO DOS CACOS } & \multirow[t]{14}{*}{ L3 } & PC63 & 79 & 16 & 4 & - & 1 & - & - \\
\hline & & PC64 & 62 & 24 & 11 & 2 & - & 1 & - \\
\hline & & PC65 & 55 & 32 & 11 & 1 & 1 & - & - \\
\hline & & PC66 & 45 & 45 & 8 & 1 & 1 & - & - \\
\hline & & PC67 & 40 & 43 & 15 & 2 & - & - & - \\
\hline & & PC68 & 38 & 54 & 5 & 2 & 1 & - & - \\
\hline & & PC69 & 52 & 34 & 11 & 1 & 1 & 1 & - \\
\hline & & PC119 & 59 & 31 & 8 & - & - & 2 & - \\
\hline & & PC122 & 53 & 38 & 7 & - & - & 2 & - \\
\hline & & PC124 & 87 & - & 13 & - & - & - & - \\
\hline & & PC126 & 88 & - & 6 & 4 & - & 2 & - \\
\hline & & PC129 & 83 & - & 15 & - & - & 2 & - \\
\hline & & PC130 & 45 & 42 & 3 & 3 & - & 1 & 6 \\
\hline & & PC131 & 43 & 49 & 5 & 3 & - & - & - \\
\hline & \multirow[t]{4}{*}{ A51c } & PC112 & 68 & - & 5 & - & - & 7 & 20 \\
\hline & & PC114 & 60 & 23 & 13 & 2 & - & 2 & - \\
\hline & & PC115 & 80 & - & 14 & 4 & - & 2 & - \\
\hline & & PC117 & 33 & - & 65 & - & - & 2 & - \\
\hline & \multirow[t]{21}{*}{ D14 } & PC91 & 58 & 30 & 8 & 4 & - & - & - \\
\hline & & PC92 & 43 & 44 & 11 & 1 & 1 & - & - \\
\hline & & PC93 & 50 & 22 & 6 & 21 & - & 1 & - \\
\hline & & PC94 & 86 & - & 12 & 1 & - & 1 & - \\
\hline & & PC95 & 49 & 43 & 7 & 1 & - & - & - \\
\hline & & PC96 & 58 & 28 & 10 & 3 & 1 & - & - \\
\hline & & PC97 & 48 & 39 & 11 & $\operatorname{tr}$ & 1 & $\mathrm{tr}$ & - \\
\hline & & PC98 & 50 & 41 & 6 & 2 & - & 1 & - \\
\hline & & PC99 & 85 & - & 14 & - & - & 1 & - \\
\hline & & PC100 & 50 & 39 & 9 & - & 1 & 1 & - \\
\hline & & PC101 & 67 & 20 & 8 & 4 & - & 1 & - \\
\hline & & PC102 & 86 & - & 13 & - & - & 1 & - \\
\hline & & PC103 & 46 & 40 & 11 & 2 & - & 1 & - \\
\hline & & PC104 & 51 & 32 & 6 & 10 & $\operatorname{tr}$ & $\operatorname{tr}$ & - \\
\hline & & PC105 & 44 & 48 & 5 & 2 & 1 & - & - \\
\hline & & PC106 & 68 & 22 & 9 & - & - & 1 & - \\
\hline & & PC107 & 85 & - & 13 & - & - & 2 & - \\
\hline & & PC108 & 50 & 35 & 11 & 3 & - & 1 & - \\
\hline & & PC109 & 42 & 48 & 6 & 3 & 1 & - & - \\
\hline & & PC110 & 63 & 21 & 12 & 3 & - & 1 & - \\
\hline & & PC111 & 48 & 41 & 7 & 2 & 1 & 1 & - \\
\hline \multirow[t]{31}{*}{ QUINTA DO ROUXINOL } & \multirow[t]{17}{*}{ A51c } & QR2 & 61 & 28 & 8 & 1 & 2 & 1 & - \\
\hline & & QR3 & 74 & 15 & 9 & 1 & 1 & - & - \\
\hline & & QR4 & 83 & - & 15 & - & - & 2 & - \\
\hline & & QR5 & 64 & 23 & 11 & 2 & - & - & - \\
\hline & & QR6 & 93 & - & 7 & - & - & - & - \\
\hline & & QR7 & 64 & 27 & 7 & - & - & 2 & - \\
\hline & & QR8 & 72 & 14 & 13 & - & 1 & - & - \\
\hline & & QR9 & 55 & 28 & 9 & 7 & 1 & - & - \\
\hline & & QR10 & 75 & 10 & 14 & - & - & 1 & - \\
\hline & & QR11 & 81 & - & 15 & 4 & - & 1 & - \\
\hline & & QR12 & 88 & - & 11 & - & - & 1 & - \\
\hline & & QR13 & 42 & 51 & 4 & 1 & 1 & 1 & - \\
\hline & & QR14 & 61 & 31 & 7 & - & 1 & - & - \\
\hline & & QR174 & 85 & - & 10 & - & - & 5 & - \\
\hline & & QR175 & 37 & 56 & 3 & - & 3 & 1 & - \\
\hline & & QR177 & 49 & 40 & 6 & 3 & - & 2 & - \\
\hline & & QR179 & 91 & - & 8 & - & - & 1 & - \\
\hline & \multirow[t]{14}{*}{ A50 } & QR41 & 88 & - & 12 & - & - & - & - \\
\hline & & QR42 & 70 & 20 & 8 & 1 & - & 1 & - \\
\hline & & QR43 & 64 & 22 & 9 & 3 & 2 & - & - \\
\hline & & QR44 & 90 & - & 10 & - & - & - & - \\
\hline & & QR45 & 64 & 27 & 6 & 2 & 1 & - & - \\
\hline & & QR46 & 57 & 31 & 9 & 2 & 1 & - & - \\
\hline & & QR47 & 79 & 11 & 10 & - & - & - & - \\
\hline & & QR48 & 88 & - & 12 & - & - & - & - \\
\hline & & QR49 & 67 & 19 & 10 & 4 & - & - & - \\
\hline & & QR50 & 45 & 46 & 6 & Trace & 2 & Trace & - \\
\hline & & QR51 & 62 & 27 & 6 & 2 & 2 & 1 & - \\
\hline & & QR153 & 87 & - & 10 & - & - & 3 & - \\
\hline & & QR161 & 33 & 56 & 7 & 2 & - & 2 & - \\
\hline & & QR167 & 42 & 50 & 5 & 2 & - & 1 & - \\
\hline
\end{tabular}




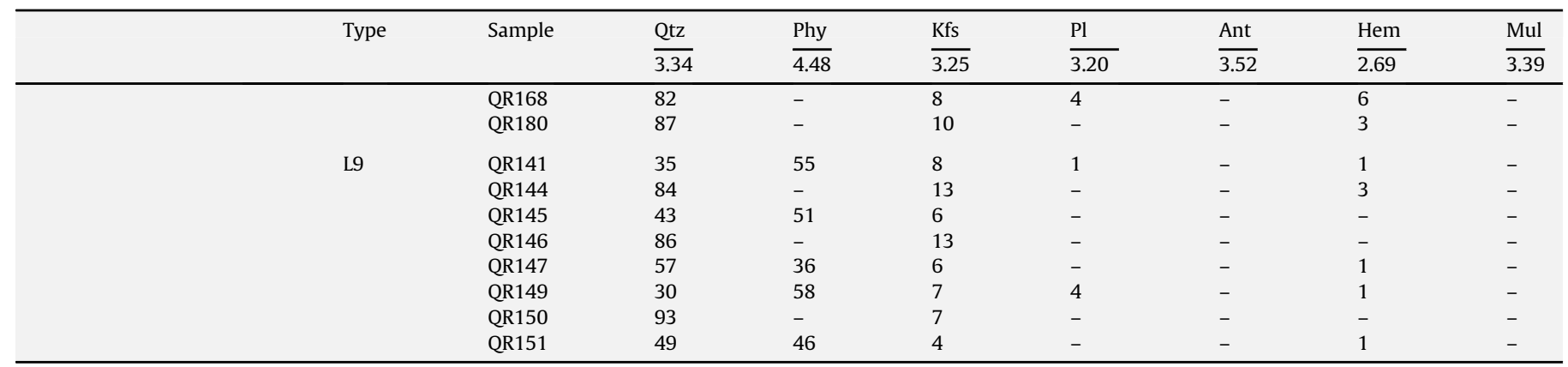

Qtz - quartz; Phy - phyllosilicates; Kfs - K-feldspar; Pl - plagioclase; Cal - calcite; Ant - anatase; Hem - hematite; Mul - mullite

and Euclidean distances, clearly shows the order and levels of clustering, as well as the distances between individual samples. It is also important to emphasize that the five sub-samples analysed from the same L9 amphorae sherd present a very high level of resemblance, of identical degree to the rest of the group, which is a very good indicator of the high-quality clustering of these compositional groups. Cluster analysis also defines the existence of three chemically distinct samples from the QR workshop. Other statistical approaches (box-and-whisker plot graphs, PCA) point to the same distinct samples, representing one sample of each analysed typology: (i) QR3 (A51c) presents upper extreme values of Ba, lower extreme values of Cs and upper outlier values of $\mathrm{Na}$; (ii) QR83 (L9) presents upper extreme values of Sb, lower extreme value of Ba and upper outlier values of Nd, Sm and Eu; (iii) QR180 (A50) presents upper extreme values of $\mathrm{Zn}$.

Initially, on the basis of typological evidence, we tried to identify eventual chemical differences according to this criterion, but all of the specimens appeared to be compositionally quite similar, and had proven almost indistinguishable in earlier studies (Cabral et al., 1993-1994; Cabral et al., 2002). Even considering some 'overlap' in the chemical classification of QR samples, because a clear separation is not to be expected in the statistical analysis of chemical data, further studies (Dias et al., 2001) emphasize the possibility of slightly differentiating L9 amphorae from those belonging to the Almagro 51c typology, especially according to the iron enrichment and the higher chemical homogeneity. On the other hand, A51c amphorae present a wider spread of data from a compositional point of view. In fact, none of the bivariate plots examined so far

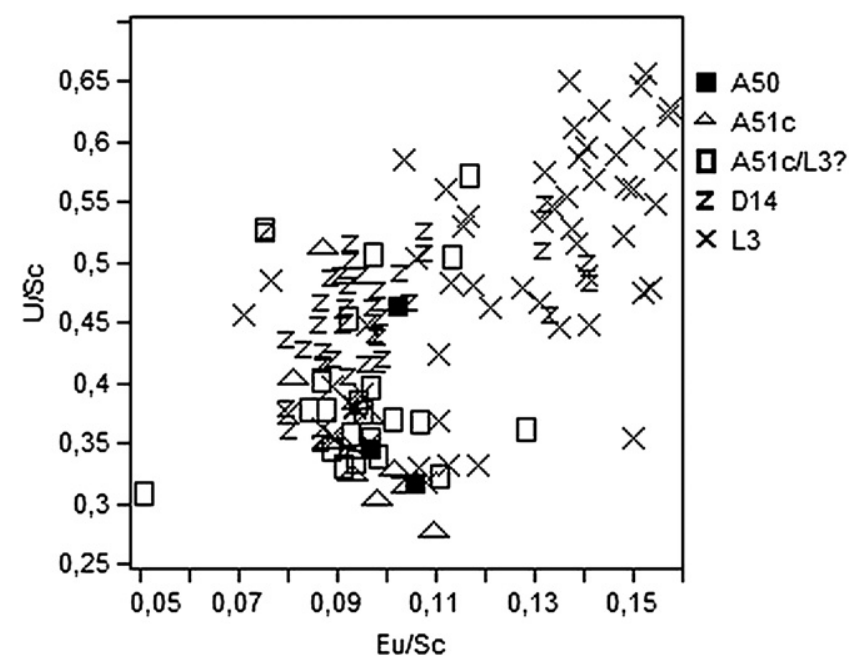

Fig. 2. Bivariate plot of Uranium versus Europium (both normalized to Sc) for the Roman amphorae from the Porto dos Cacos workshop, according to the typological classification. convincingly distinguished QR typologies from each other, but we observed that, with the exception of a few L9 samples, the other L9 fragments were indeed distinguishable, as shown in Figs. 4 and 5, especially due to higher contents of Fe, as well as lower contents of REE, Ta, Hf, Th and Na.

From an archaeological point of view, this is an acceptable hypothesis, identifying a more heterogeneous chemical composition for A51c samples, especially as compared to L9. This was probably due to a longer period of production, allowing for a greater need to use diverse outcrop exploitations of clay raw materials.

\subsection{Porto dos Cacos and Quinta do Rouxinol workshops: a path to their differentiation}

The goal of the statistical analysis employed in this work is to isolate and refine a reference group for pottery production at each production centre (PC and $\mathrm{QR}$ ) for further comparison to consumption centres, and also other kilns, in order to allow a better differentiation between them and to establish a geochemical signature for each workshop.

Several statistical approaches were carried out to this purpose, using both absolute and normalized values, coupled with diverse analyses of geochemical data, namely trace element data, in accordance with their geochemical behaviour and distribution by geo-environment.

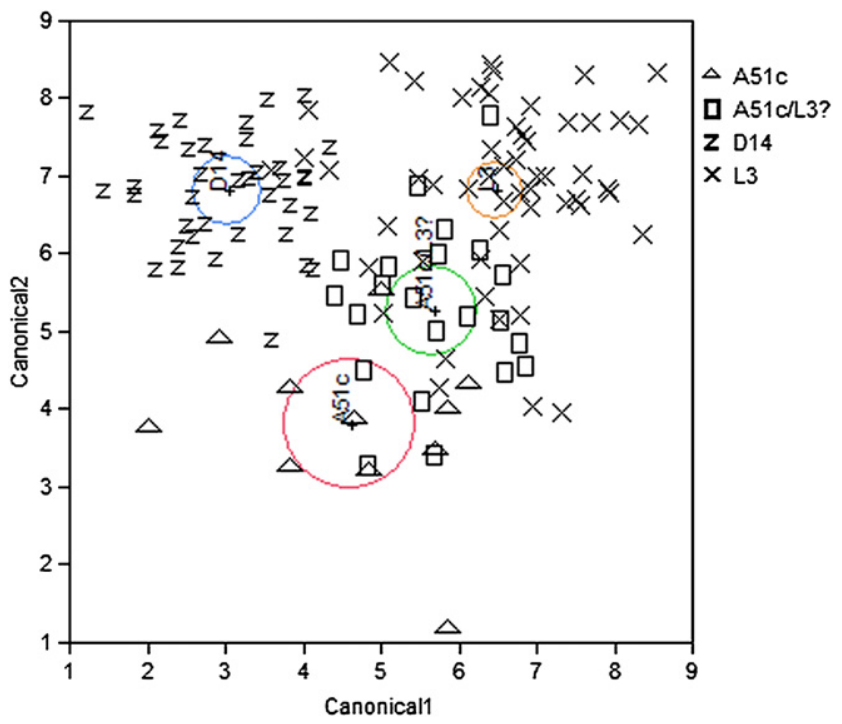

Fig. 3. Biplot showing amphorae samples from Porto dos Cacos on canonical roots 1 and 2 (chemical data normalized to Sc). Normal 50\% contours are visible, containing roughly $50 \%$ of the points for that group. 


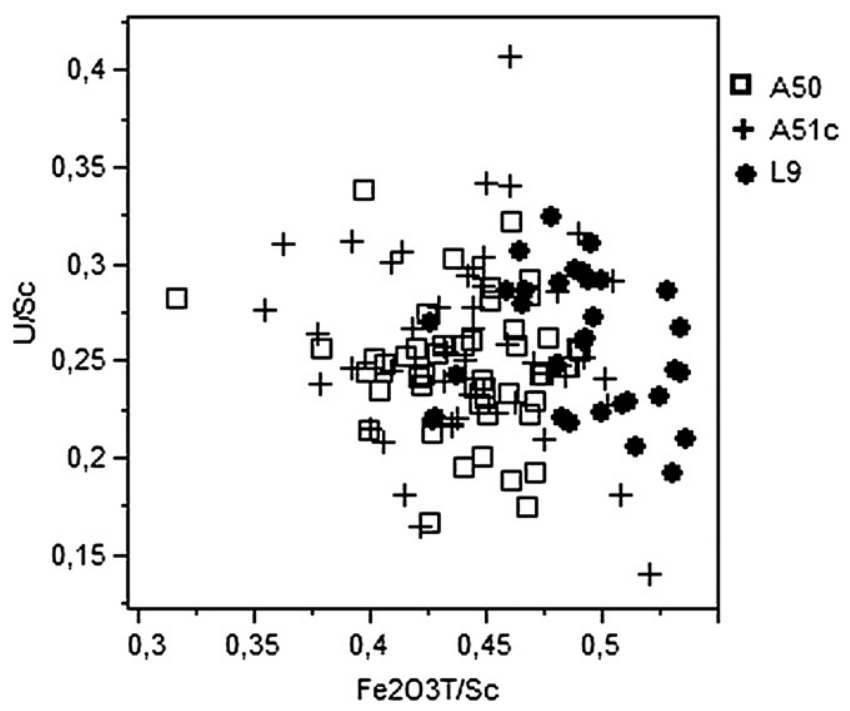

Fig. 4. Bivariate plot of Uranium versus Iron (both normalized to Sc) for the Roman amphorae from the Quinta do Rouxinol workshop, according to the typological classification.

Considering all the amphorae sherds analysed for each workshop, the resulting dendrogram using Ward's hierarchical clustering method and Euclidean distances suggests the existence of five outliers from the PC workshop (PC2, PC17, PC19, PC40, PC123) and two from the $\mathrm{QR}$ workshop (QR3, QR180), displaying the features mentioned above, with the exception of QR83, which also presented the lowest parting degree.

After disregarding these seven outlier samples, a new statistical approach has been taken in order to better define compositional groups. A first approach, also by cluster analysis applied to chemical elements normalized to Sc as variables, using Ward's amalgamation rule and the Pearson correlation coefficient, suggests the existence of two clusters (Fig. 6), both comprising samples from both the PC and QR workshops. Nevertheless, they are well separated, each sub-group including samples from only one workshop. The pottery

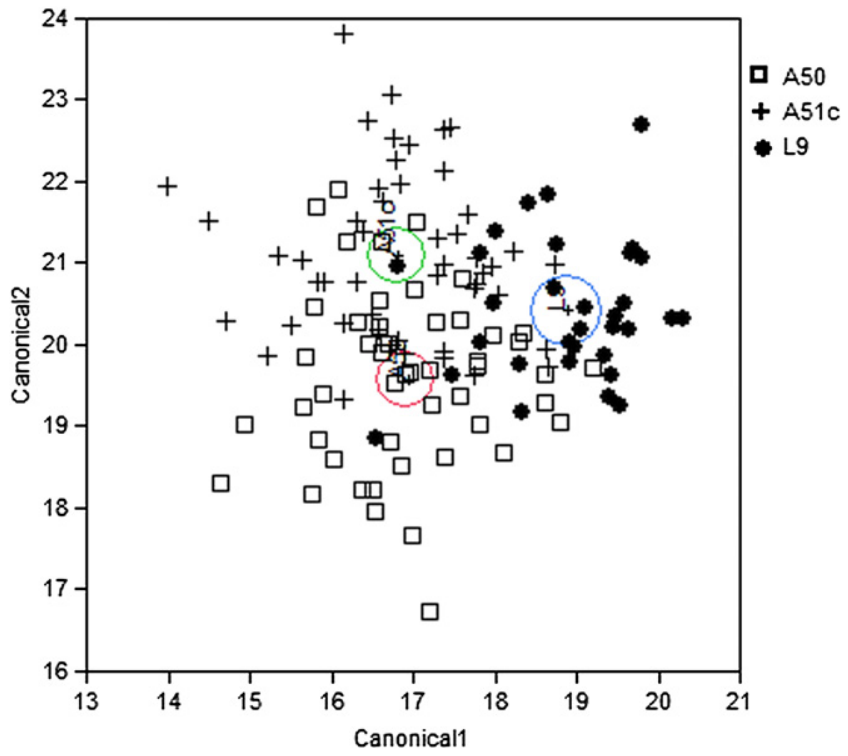

Fig. 5. Biplot showing amphorae samples from Quinta do Rouxinol on canonical roots 1 and 2 (chemical data normalized to Sc). Normal 50\% contours are visible, containing roughly $50 \%$ of the points for that group.

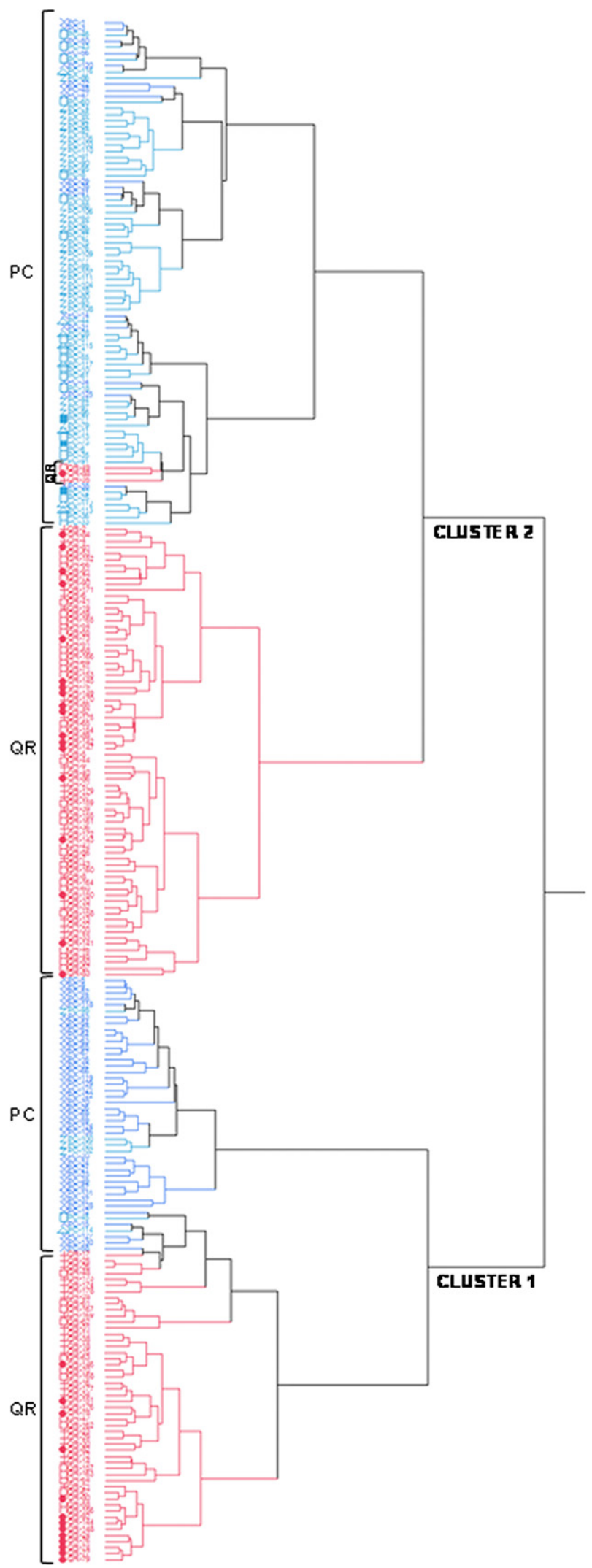

Fig. 6. Classification of amphorae from Porto dos Cacos and Quinta do Rouxinol considering typological taxonomy. The hierarchical cluster analysis was applied to chemical elements (normalized to Sc) using Ward's amalgamation rule and the Pearson correlation coefficient 
from the two sites indicates clear intra- and inter-site interactions. There appears to be an intra-site distribution, as the presence of sub-groups within the ceramics from these two workshops implies the use of more than one clay source, and, at the same time, the existence of clusters comprising samples from both sites indicates the natural geochemical heterogeneity of this estuary basin. It is important to emphasize that some correlation with the typology has been established, again more evident for PC amphorae, as cluster 1 comprises mainly PC samples of L3 shape (Fig. 6). The other sub-group of cluster 1 comprises $50 \mathrm{QR}$ samples of various typologies, and all the remaining samples are included in cluster 2 , but with a clear distinction between PC and QR workshops (only samples QR 49 - A50, QR 88 - L9 and QR 35 - A51c are mixed within the PC sub-group of cluster 2; the other sub-group of cluster 2 only contains QR samples). It is important to underline the difficulty of assigning with certainty PC samples to the PC workshop and QR samples to the QR workshop by using this statistical approach, since, as mentioned above, some PC samples group with QR samples.

Principal component analysis (PCA) is one of the most frequently used techniques for exploring underlying relationships in multivariate data. Canonical discriminant analysis (CA) consists of finding a linear combination which gives the maximum ratio of the variability between groups applicable to the variability within groups. A PCA of the standardized chemical data identified the seven chemical outliers already suggested by cluster analysis. The seven outliers were subsequently omitted from all further analyses, to allow formal comparisons between different plots. Thus, we have repeated the PCA (Fig. 7) and CA (Fig. 8) analyses after this omission, and results are labelled according to whether the samples belong to the PC workshop or the QR workshop, also considering the typological classification. Following a principal components analysis that selected four components accounting for $70.79 \%$ of the original variation, two well-defined groups were mapped into the components space, each belonging to a specific workshop, especially considering factors 1, 2 and 3 (accounting for $64.80 \%$ of the original variation). In terms of the first principal component of the loading plots, lanthanides, actinides and $\mathrm{Hf}, \mathrm{Zr}$, Ta display large positive loadings, while $\mathrm{Sb}$ and $\mathrm{Fe}$ have a negative weight, and $\mathrm{Rb}$, $\mathrm{Zn}, \mathrm{K}, \mathrm{Cs}, \mathrm{Ba}, \mathrm{Cr}$, As have a very low positive weight. On the other hand, the second component clearly separates a set of elements, with large negative loadings, from the one including $\mathrm{Cr}, \mathrm{Co}, \mathrm{As}, \mathrm{Ba}$ and $\mathrm{U}$, and from another with positive loadings comprising $\mathrm{K}, \mathrm{Fe}$, $\mathrm{Rb}, \mathrm{Cs}, \mathrm{Zn}, \mathrm{Sb}$ and Ta. The corresponding score plots show two groups (the outlier samples were omitted), each one corresponding to one of the workshops. The PC workshop is characterized by high concentrations of $\mathrm{Co}$, As and $U$ and low concentrations of the elements presenting large positive loadings on the second component ( $\mathrm{Fe}, \mathrm{Zn}, \mathrm{Sb}, \mathrm{Rb}, \mathrm{K}$ ); the other group, comprising $\mathrm{QR}$ workshop amphorae, shows instead large concentrations of Fe and $\mathrm{Sb}$, and also of $\mathrm{Rb}$ and $\mathrm{Zn}$. None of the two workshops shows a particular composition with respect to rare earth elements. Two robust clusters of the major sites are also clearly visible in the CA analysis (Fig. 8), corresponding to the samples from the PC and QR workshops.

Bivariate plots (after normalization to Sc) were also sometimes sufficient to reveal patterns in the chemical data, especially if they include iron and uranium (Fig. 9), as well as antimony and zinc,

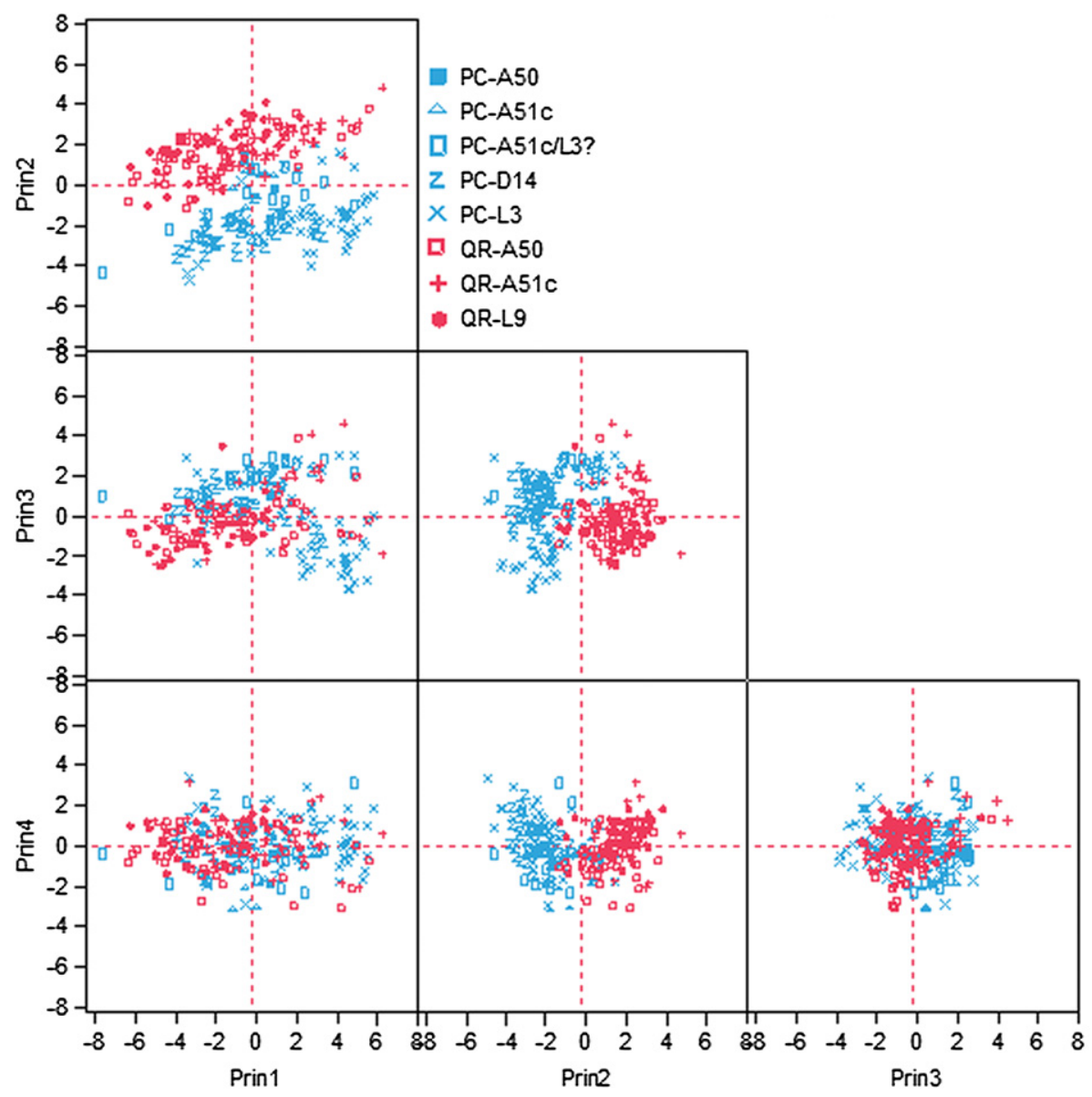

Fig. 7. A two-dimensional component plot based on PCA of the PC and QR chemical data (normalized to Sc), omitting seven outliers (five from PC and two from QR). 


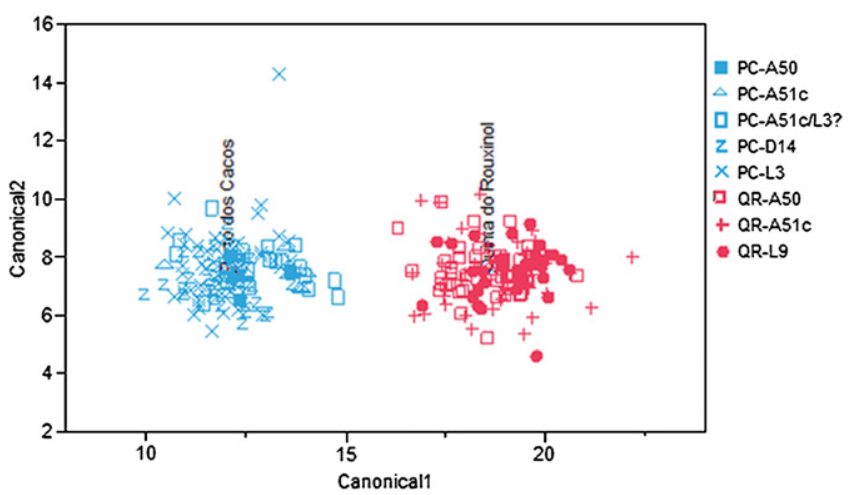

Fig. 8. A two-dimensional canonical plot based on $C A$ of the $P C$ and $Q R$ chemical data (normalized to Sc), omitting seven outliers (five from PC; two from $\mathrm{QR}$ ).

antimony and uranium, arsenic and uranium, iron and zinc. According to these patterns, certain location-specific reference sets were defined, and the members of each set were ceramic specimens from each workshop.

In terms of the mineralogical composition obtained by XRD, quartz is the dominant mineral phase in almost all samples, particularly in QR analysed sherds (Table 3). The XRD analyses revealed, in addition to a great quantity of quartz, lesser amounts of mica (the only phyllosilicate detected) and K-feldspar, and finally plagioclase, hematite and anatase in trace amounts. The presence of mullite was only detected in two samples from PC kilns. XRD provided the key to the reconstruction of the firing temperatures that varied around $900{ }^{\circ} \mathrm{C}$, and were higher than $1000^{\circ} \mathrm{C}$ only in two cases. The range of the firing temperatures was determined according to the stability of mica components and the newly formed mineral phase of mullite, which is encountered when aluminous clay minerals are heated to temperatures of $1000^{\circ} \mathrm{C}$ and above. Thus, the XRD data indicated relatively high firing temperatures, with some variability due to a few low-fired fragments and a very limited number of samples fired at temperatures higher than $1000{ }^{\circ} \mathrm{C}$.

As a result, as expected considering the geological context of the Tagus estuary, the mineralogical composition obtained by XRD does not differentiate the two workshops, reflecting once again the natural inhomogeneity of the raw material, intrinsic to the

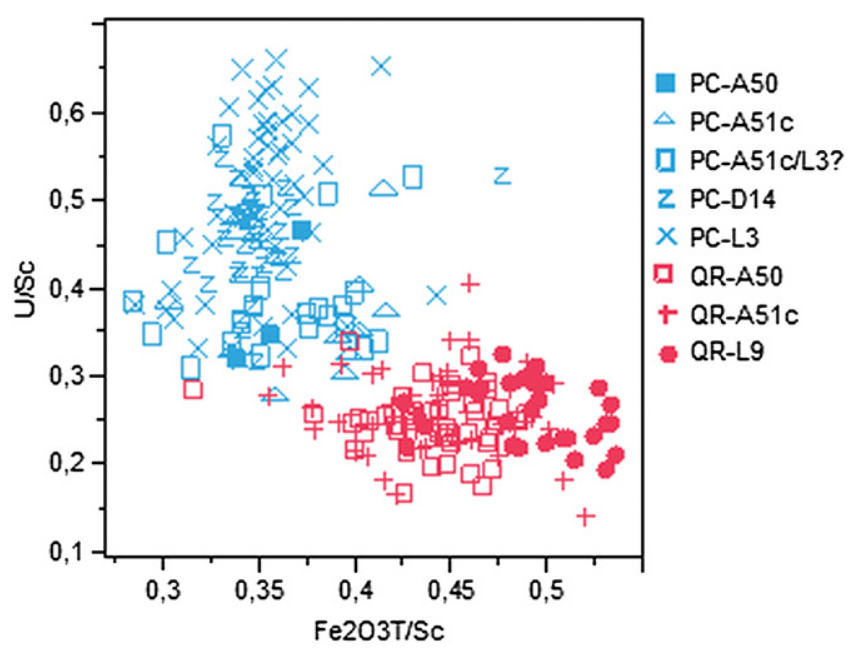

Fig. 9. Bivariate plot of Uranium versus Iron (both normalized to Sc) for amphorae from the two workshops (PC and QR). sedimentary basin, with inputs belonging to diverse geological contexts.

The chemical variability within the PC workshop points to raw materials presenting a general enrichment of $\mathrm{Co}$, As and U. Cobalt concentrations usually reflecting the abundance of mafic minerals are common in very fine-grained mudrocks, arsenic is usually concentrated in clays, hydrous Fe and Mn oxides, sulphides and phosphates, and uranium is closely related to redox conditions, representing the $U$ enrichment attributed to oxidation-reduction processes. Thus, the high contents of these three elements may be related to the mixture of raw materials with brackish clays, as already noticed for the Zambujalinho workshop in the Sado basin (Prudêncio et al., 2009), where the high contents of $U$ were associated with the organic matter present in the sediments of that estuarine environment.

In the QR workshop, the paste of the amphorae is particularly enriched in $\mathrm{Fe}$ and $\mathrm{Sb}$ (and also $\mathrm{Rb}$ and $\mathrm{Zn}$ ). Fine-grained argillaceous and organic-rich sediments are typically enriched in antimony relative to their parent lithologies, reflecting the tendency for the element to become absorbed by hydrous oxides, organic residues and clay minerals in favourable environments (Ure and Berrow, 1982). The abundance of iron in sedimentary environments is determined by various factors, such as origin, being generally enriched in mafic rocks relative to felsic. Secondary hydrous oxides represent the dominant Fe phase, and there is a tendency for these hydrous Fe phases to form surface oxide coatings, reflecting a direct relationship between the total $\mathrm{Fe}$ content and the specific surface areas of particles (Ure and Berrow, 1982), consequently resulting in clays generally enriched in this element.

\section{Conclusions}

By providing geochemical reference groups for the $\mathrm{PC}$ and $\mathrm{QR}$ Roman production centres, with fine differentiation between them, the obtained results contribute significantly to our understanding of the nature of amphora production on the lower Tagus. This is especially important because the base raw materials at both sites are of a similar type, and the fine discrimination relies mainly on trace elements compositional differences revealed by INAA and related geochemical /chemometric approaches.

From a technological perspective, this work allowed us to estimate the firing temperatures of the amphorae, confirming the relatively high temperatures reached by these kiln types. The presence/absence and abundance of high-temperature phases confirm the indications pertaining to the temperatures reached inside the kiln, and the presence of mica has been shown to indicate firing temperatures lower than $900^{\circ} \mathrm{C}$. Another important technological aspect is the evidence of the mixture of raw materials with brackish clays in the PC workshop, as pointed out by the high contents of $\mathrm{Co}$, As and $\mathrm{U}$; also, in the QR workshop, the enrichment in $\mathrm{Fe}$ and $\mathrm{Sb}$ presented by the paste of amphorae seems to indicate the use of fine-grained argillaceous and organic-rich sediments.

This study of lower Tagus Roman ceramic production centres that sought to establish the geochemical fingerprints of each centre will be useful in further works of provenance and sourcing studies (comparison with consumption centres and with other regional /trans-regional workshops), contributing to the ascertainment of merchandise distribution patterns in Roman trade and their impact in Lusitania.

Once a framework for reference has been established, significant aspects related to pottery production and to the regional and imperial economy (in terms of production, distribution and consumption) will become clearer, thus confirming the potential of 
this type of study for understanding the social and economic history of Antiquity.

Ultimately, we may also revise our perception of the model of this type of kiln workshops, clarifying their function not only as simple production centres, but as veritable poles of regional economic development. This will also make possible clarifying the relationships and the levels of interdependence between pottery centres and the fish salting and transforming units related to them at a regional scale.

\section{Acknowledgements}

The authors would like to thank the anonymous referees for their constructive comments and suggestions. We also give very special thanks to Raul Carstocea, UCL Institute of Archaeology, who carefully revised the English language of this manuscript, as well as to Thilo Rehren for his editorial efforts.

\section{References}

Biscaye, P.E., 1965. Mineralogy and sedimentation of recent deep-sea clay in the Atlantic Ocean and adjacent seas and oceans. Geological Society of America Bulletin 76, 803-832.

Cabral, J.M.P., Gouveia, M.A., 1984. Sobre a proveniência de ânforas e outra cerâmica comum lusitano-Romana de Tróia de Setúbal. Arqueologia e História, Série X (I) 143-153.

Cabral, J.M.P., Gouveia, M.A., Morgado, I., 1993-1994. Caracterização química das produções de ânforas do Vale do Tejo: II - Quinta do Rouxinol. Conimbriga, XXII-XXXIII, 191-200.

Cabral, J.M.P., Gouveia, M.A., Morgado, I., 1996. Caracterização química das produções de ânforas do Vale do Tejo: I - Porto dos Cacos. In: CMS \& Pub. D. Quixote, Actas das Primeiras Jornadas sobre a Romanização dos Estuários do Tejo e do Sado, pp. 301-322.

Cabral, J.M.P., Fonseca, S.M., Gouveia, M.A., 2000. Caracterização química das produções de ânforas do Sado: I - Oficina do Pinheiro. In: Contributos das Ciências e das Tecnologias para a Arqueologia da Península Ibérica - Actas do 3 ${ }^{\circ}$ Congresso de Arqueologia Peninsular, vol. IX. ADECAP, Porto. 141-160.

Cabral, J.M.P., Fonseca, S.M., Gouveia, M.A., 2002. Caracterização química das produções de ânforas do Vale do Tejo: III - Quinta do Rouxinol (continuação). Instituto Português de Arqueologia. Revista Portuguesa de Arqueologia 5 (2), 325-338.

Dias, M.I., Prudêncio, M.I., Raposo, J., Gouveia, M.A., Fabião, C., Guerra, A., Bugalhão, J., Duarte, A.L., Sabrosa, A., 2001. Caracterização química por AAN das formas de ânfora de um centro de produção do estuário do Tejo: Quinta do Rouxinol (Portugal). In: Actas do IV Congreso Nacional de Arqueometria, Valencia, pp. 88-93.

Dias, M.I., Prudêncio, M.I., Rocha, F., 2003. Applied study of cultural heritage and clays. In: Pérez-Rodríguez, José Luis (Ed.), Sociedade Española de Arcillas (SEA). CSIC, Madrid, pp. 187-200.

Dias, M.I., Prudêncio, M.I., 2007. Neutron activation analysis of archaeological materials: an overview of the ITN NAA laboratory, Portugal. Archaeometry 49 (2), 383-393.

Dias, M.I., Prudêncio, M.I., 2008. On the importance of using scandium to normalize geochemical data preceding multivariate analyses applied to archaeometric pottery studies. Microchemical Journal 88, 136-141.

Duarte, A.L.C., 1990. Quinta do Rouxinol: a produção de ânforas no vale do Tejo. In: Ânforas Lusitanas: tipologia, produção, comércio (eds. A. Alarcão and F. Mayet),
Museo Monográfico de Conímbriga/Diff.E. de Boccard, Conímbriga, Paris, pp. 96-115

Duarte, A.L.C., Raposo, J.M.C., 1996. Elementos para a Caracterização das Produções Anfóricas da Quinta do Rouxinol (Corroios/Seixal). In: Filipe, Gr., Raposo, Jorge M.C. (Eds.), Ocupação Romana na Margem Esquerda dos Estuários do Tejo e do Sado. Nova Enciclopédia, vol. 53. Publicações Dom Quixote/Câmara Municipal do Seixal, Lisboa, pp. 237-247.

Fabião C., 2004. Centros oleiros da Lusitania: balanço dos conhecimentos e perspectivas de investigação. Actas del Congreso Internacional Figlinae Baeticae. Talleres alfareros y producciones cerámicas en la Bética Romana (ss. II a.C-VII d.C.), pp. 379-410.

Fabião, C., 2008. Las Ânforas de Lusitânia. In: Bernal Casasola, D., Ribera i Lacomba, A. (eds.). Cerâmicas Hispanorromanas. Un estado de la cuestión. Cádiz: Universidad de Cádiz, pp. 725-745.

Gouveia, M.A., Prudêncio, M.I., Freitas, M.C., Martinho, E., Cabral, J.M.P., 1987. Interference from uranium fission products in the determination of rare earths, zirconium and ruthenium by instrumental neutron activation analysis in rocks and minerals. Journal of Radioanalytical Radiochemistry, Articles 114(2), 309-318.

Govindaraju, K., 1994. Geostandards Newsletter 18 (Special Issue), 1.

Guerra, A., 1996. Marcas de Ânfora Provenientes do Porto dos Cacos (Alcochete). In: Filipe, Gr., Raposo, Jorge M.C. (Eds.), Ocupação Romana na Margem Esquerda dos Estuários do Tejo e do Sado. Nova Enciclopédia, vol. 53. Publicações Dom Quixote/Câmara Municipal do Seixal, Lisboa, pp. 267-282.

Martinho, E., Gouveia, M.A., Prudêncio, M.I., Reis, M.F., Cabral, J.M.P., 1991. Factor for correcting the ruthenium interference in instrumental neutron activation analysis of barium in uraniferous samples. Applied Radiation and Isotopes 42 (11), 1067-1071

Martín-Pozas, J.M., 1968. El analisis mineralógico cuantitativo de los filosilicatos de la arcilla pordifracción de rayos X. PhD thesis, Univ. Granada, Spain.

Mayet, F., Schmitt, A., Silva, C.T., 1996. Les amphores du Sado (Portugal). Prospection des fours et analyse du matériel. Diff. E. De Boccard, Paris.

Prudêncio, M.I., Dias, M.I., Raposo, J., Gouveia, M.A., Fabião, C., Guerra, A., Bugalhão, J., Duarte, A.L., Sabrosa, A., 2003. Chemical characterisation of amphorae from the Tagus and Sado estuaries production centres (Portugal). In: Di Pierro, S., Serneels, V., Maggetti, M. (Eds.), Ceramic in the Society, Proceedings of the EMAC'01, Fribourg, pp. 245-253.

Prudêncio, M.I., Dias, M.I., Gouveia, M.A., Marques, R., Franco, D., Trindade, M.J., 2009. Geochemical signatures of Roman amphorae produced in the Sado River estuary, Lusitania (Western Portugal). Journal of Archaeological Science 36, $873-883$.

Raposo, J.M.C., 1990. Porto dos Cacos: uma oficina de produção de ânforas romanas no vale do Tejo. In: Alarcão, A., Mayet, F. (Eds.), Ânforas Lusitanas: tipologia, produção, comércio. Museo Monográfico de Conímbriga/Diff.E. de Boccard, Conímbriga, Paris, pp. 117-151.

Raposo, J.M.C., Sabrosa, A.J.G. and Duarte, A.L.C., 1995. Ânforas do Vale do Tejo: as olarias da Quinta do Rouxinol (Seixal) e do Porto dos Cacos (Alcochete). Trabalhos de Antropologia e Etnologia. Porto, vol. 35(3), pp. 331-352 (Actas do $1{ }^{\circ}$ Congresso de Arqueologia Peninsular, vol. VII).

Raposo, J.M.C., Duarte, A.L.C., 1996. O Forno 2 do Porto dos Cacos (Alcochete). In: GrFilipe, Raposo, Jorge M.C. (Eds.), Ocupação Romana na Margem Esquerda dos Estuários do Tejo e do Sado. Nova Enciclopédia, vol. 53. Publicações Dom Quixote/Câmara Municipal do Seixal, Lisboa, pp. 249-266.

Raposo, J., Fabião, C., Guerra, A., Bugalhão, A.L., Sabrosa, A., Dias, M.I., Prudêncio, M.I., Gouveia, A., 2005. OREsT Project: Late Roman pottery productions from the Lower Tejo. In: Gurt i Esparraguera, J.M., Buxeda i Garrigós, J., Cau Ontiveros, M.A. (Eds.), LRCW I, Late Roman Coarse Wares, Cooking Wares and Amphorae the Mediterranean. Archaeology and Archaeometry, BAR International Series, vol. 1340, pp. 37-54.

Schultz, L.G., 1964. Quantitative interpretation of mineralogical composition X-ray and chemical data for the Pierre Shale. Geological Survey 391.

StatSoft, Inc., 2008. STATISTICA (data analysis software system), version 8.0. www. statsoft.com.

Ure, A.M., Berrow, M.L., 1982. The elemental constituents of soils. In: Environmental Chemistry, vol. 2. Royal Society of Chemistry, London, pp. 94-204. 